\title{
Semantic Processing Without Conscious Identification: Evidence From Event-Related Potentials
}

\author{
Georg Stenberg, Magnus Lindgren, Mikael Johansson, Andreas Olsson, and Ingmar Rosén \\ University of Lund
}

\begin{abstract}
Three event-related potential (ERP) experiments examined whether semantic content can be accessed from visually presented words that cannot be consciously identified. Category labels were shown to participants, followed by masked, briefly exposed words that were either exemplars of the category or not exemplars. The task was to verify the category, by guessing if necessary, and to identify the word, naming it if possible. Exposure durations were selected to allow identification in approximately half the trials. For identified words, there was a marked difference in the ERP response between in-category and out-ofcategory words because of an N400 component. For unidentified words, there was a similar although smaller difference. Conscious identification was defined using a variety of approaches: verbal report, 6-alternative forced choice, and binary categorization (in the context of the regression method; A. G. Greenwald, M. R. Klinger, \& E. S. Schuh, 1995). By any definition, ERPs for unidentified words showed evidence of semantic processing. In addition, there were differences in the neuronal populations recruited to process above-threshold versus below-threshold words, suggesting qualitative differences.
\end{abstract}

Perception of stimuli under severely data-limited conditions has been studied throughout the history of experimental psychology, and a number of early studies (reviewed by Adams, 1957) showed that participants performed at levels exceeding chance while expressing very low subjective confidence in their perceptions. The dissociation between a direct measure of aware perception, such as a verbal report, and a different measure, such as a forced guess (which is purportedly sensitive to unconscious processing), has remained a focus of research interest. Whereas the early studies often focused on sensory judgments about nonsymbolic stimuli such as weights and lines, Marcel and others (Allport, 1977; Balota, 1983; Fowler, Wolford, Slade, \& Tassinary, 1981; Marcel, 1980, 1983a, 1983b; Marcel \& Patterson, 1978) set the agenda for much subsequent research by the use of semantic word processing as the measure of interest and by the choice of visual masking as the means to prevent awareness.

However, studies of Marcel's type have been subjected to methodological criticism, both concerning procedural and conceptual

Georg Stenberg, Mikael Johansson, and Andreas Olsson, Department of Psychology, University of Lund, Lund, Sweden; Magnus Lindgren, Department of Psychology, University of Lund, and Department of Clinical Neuroscience, University of Lund; and Ingmar Rosén, Department of Clinical Neuroscience, University of Lund.

This study was supported by a grant from the Swedish Council for Research in the Humanities and the Social Sciences (HSFR). Parts of this research (Experiment 2) were presented at the IOP Conference, 1998 in Taormina, Italy. We are grateful to Carina Berglund for running Experiment 3 and constructing its stimulus material and to Aleksandra Walfisz for skillful assistance with electrophysiological data collection. We are also indebted to Phil Merikle, Phillip Holcomb, and two anonymous reviewers for helpful comments on an earlier version of this article.

Correspondence concerning this article should be addressed to Georg Stenberg, who is now at Department of Social Sciences, Växjö University, SE-35195 Växjö, Sweden. Electronic mail may be sent to Georg. Stenberg@psychology.lu.se. issues (Holender, 1986). One aspect of this criticism concerns the implied concept of a threshold (i.e., a quantity of stimulus energy, duration, etc.), below which no stimulus can give rise to conscious experience. Once a threshold has been determined, the experimental procedure proceeds to present stimuli below this level, on the basis of the assumption that they will remain unconscious. The threshold concept has been questioned and replaced in theoretical discussions by signal-detection theory's stochastic concept (Green \& Swets, 1974). Pragmatically, the determination of a threshold raises difficulties, because a threshold determined at the start of a session may not remain valid during the whole session because of adaptation processes (Holender, 1986).

Much research has been aimed at documenting a dissociation between unconscious and conscious processing by showing the former in operation without the latter. Conceptually, this dissociation paradigm rests on the comparison between a measure of conscious processing, supposedly showing zero sensitivity, and a measure of unconscious processing, simultaneously showing evidence of above-zero sensitivity (Cheesman \& Merikle, 1986; Merikle \& Reingold, 1992). Disagreements about suitable candidates for these measures abound.

The measure of unconscious processing is often referred to as the indirect measure, because many recent studies have used the indirect effect of a masked stimulus (the prime) on the processing of a subsequent stimulus (the target) as the indicator of unconscious processing. This method has the advantage of being driven by an involuntary, automatic effect. It has the drawback of being sensitive to the time gap between prime and target, across which the prime is supposed to exert its effect. Some recent studies have suggested that the effect may be extremely ephemeral and clearly detectable only at time spans less than $200 \mathrm{~ms}$ (Greenwald, Draine, \& Abrams, 1996; see, however, Balota, 1983; Fowler et al., 1981).

The choice of the measure of conscious processing, the direct measure, is even more controversial: It concerns the method for securing negative evidence of stimulus identification. The discus- 
sion on this topic has benefited from conceptual clarification (Cheesman \& Merikle, 1986; Merikle \& Reingold, 1992; Reingold \& Merikle, 1990). If the demarcation line is drawn by the participant's verbal report, the study is said to use a subjective threshold. If, on the other hand, a performance test defines identification, often by using presence-absence determination as the task and signal-detection sensitivity as the measure, then the study is said to rely on an objective threshold (Cheesman \& Merikle, 1986; Merikle \& Reingold, 1992; Reingold \& Merikle, 1990).

The choice that is supported by the longest tradition is a verbal report from the experimental participant. It has the great advantage of self-evident face validity, because what is meant by conscious processing in ordinary discourse is surely that which is accessible to introspection. However, in an experimental context, verbal report has the serious disadvantage that a fleeting or partial awareness of the stimulus may be judged irrelevant by the participant and therefore not be adequately communicated to the experimenter.

A binary-choice task has become the preferred alternative, which often focuses on the presence or absence of a stimulus in an assigned time slot of the trial. A binary choice allows the computation of $d^{\prime}$ as a measure of sensitivity, independent of response bias. Also, a binary-choice draws on every vestige of conscious processing, because in mustering the available evidence before choosing a response, the participant can ideally use partial and fleeting impressions, including those not accessible to a verbal report.

In discussing these alternatives, Reingold and Merikle (1990) formulated two criteria, both of which are desirable but not easily reconciled with each other. One is the exhaustiveness criterion: The test of stimulus identification (the direct test) should encompass all forms of conscious processing relevant to the task. This is the respect in which a subjective threshold is lacking; the verbal report given by the participant may leave out such thoughts and perceptions as he or she finds irrelevant or too uncertain to report. The participant sets the criterion for what is worth reporting, not the experimenter, and by this token, a highly desirable feature of experimental control is sacrificed (Eriksen, 1960).

The exclusiveness criterion focuses on what the direct test should not encompass: It should be sensitive only to conscious processing, not to putative forms of nonconscious processing. The latter should be the province of the indirect test, which is designed to measure nonconscious processing, if any exists. If the direct test encroaches on its domain, there may be nothing left for the indirect test to measure. If the participant draws on a subjectively experienced response tendency, which is the end result of inaccessible, unconscious processing, then the direct measure becomes contaminated by unconscious processing, Thus, if a presence-absence judgment (forming the direct task) is sensitive to nonconscious influences to the same degree as the semantic or lexical judgment that forms the indirect task, then demonstrations of these nonconscious processes will forever elude researchers, even if they exist. Reducing stimulus energy or duration to a level at which the direct measure indicates zero sensitivity may demand such a draconian reduction that the phenomenon is effectively defined out of existence.

The two criteria serve different functions, reminiscent of the procedures meant to protect against Type I and Type II errors in statistical inference. The exhaustiveness criterion is designed to make demonstrations of unconscious processing reliable, and the exclusiveness criterion is designed to make them possible. Caught on the horns of this dilemma, no direct measure has proved entirely satisfactory. In a strategy of converging evidence, this study used several alternative direct measures.

\section{A Psychophysiological Indicator}

The purpose of the present study was to elucidate the question of unconscious processing by introducing a different dependent variable to gauge its effects. To replace the indirect measure of previous studies of semantic priming, we used a psychophysiological indicator of semantic processing. The task involved semantic categorization. The participant was presented with a category label (e.g., "mammal"), followed by a masked, briefly exposed word that may (e.g., "horse") or may not (e.g., "apple") belong to the cued category. Variation in exposure duration ensured that some of the words would be identified by the participant and some not. We instructed participants to determine whether the word belonged to the category, guessing if necessary, and to read the word aloud, if possible. Event-related potentials (ERPs) were recorded along with each presentation of a masked word.

A component of the electrophysiological response of the brain has been shown to be sensitive to semantic processing. In a seminal study, Kutas and Hillyard (1980) first identified this component within a sentence-processing paradigm. By focusing on the final words in sentences that were visually presented word by word, the authors found that words deviating from their preceding context (e.g., "She spread the warm bread with socks") evoked a response different from words conforming to it (e.g., "She spread the warm bread with butter"). Identified by its latency and its negative polarity, the component was named the $\mathrm{N} 400$.

It is important that the N400 is specifically sensitive to semantic processing. The N400 is not elicited by physical deviations (e.g., words appearing in an unexpected font or letter size). Outside of the original sentence paradigm, the $\mathrm{N} 400$ has also been identified in experiments using single words or word pairs as stimuli, with tasks such as lexical decision (Bentin, McCarthy, \& Wood, 1985), judgments of semantic relatedness (Bentin, 1987), or category membership (Polich, 1985). Further support for the semantic nature of the processing that gives rise to the $\mathrm{N} 400$ is that it can be elicited by other meaning-carrying stimuli, such as pictures (Barrett \& Rugg, 1990; Holcomb \& McPherson, 1994; Nigam, Hoffman, \& Simons, 1992) or sign-language gestures (Neville et al., 1997).

Although N400 effects have been demonstrated in semantic priming paradigms, the underlying processes have not been fully identified. Some researchers have suggested that the component is automatically moderated by the process of spreading activation (Fischler \& Raney, 1991), whereas others have proposed that it is determined by controlled processing, wholly or in part (Osterhout \& Holcomb, 1995). Behavioral research has identified three mechanisms that contribute to semantic priming: automatic spreading activation, expectancy, and postlexical checking (Neely, 1991; Neely \& Keefe, 1989). All three may play a part in generating the $\mathrm{N} 400$, although discussions have tended to simplify the possibilities to a contrast between automatic (the first mechanism) and strategic processing (the other two). Evidence in favor of the view that automatic processing is at work is the fact that the effect is 
found at short SOAs (Besson, Fischler, Boaz, \& Raney, 1992; Boddy, 1986) and even with simultaneous presentation of prime and target (Anderson \& Holcomb, 1995). The N400 effect occurs when the task is orthographic (i.e., nonsemantic; Besson et al., 1992; Kutas \& Hillyard, 1989) and when the proportion of related prime-target pairs is low (Holcomb, 1988). Recently, the N400 to auditorily presented words has been demonstrated even in sleep (Brualla, Romero, Serrano, \& Valdizan, 1998). On the other hand, the N400 presupposes focused spatial attention (McCarthy \& Nobre, 1993), and it does not interact with stimulus degradation (Holcomb, 1993) as would be expected of a prelexical effect. It is influenced by expectancy (Bentin, 1987), and it is larger in an attention-promoting condition than in a low-attention condition (Holcomb, 1988) and earlier at long than at short SOAs (Anderson \& Holcomb, 1995).

Most pertinent for our present purposes, two studies with masked primes (Brown \& Hagoort, 1993; Münte \& Heinze, 1991) have failed to find $\mathrm{N} 400$ effects for the target words, although there was reaction-time priming in one of these studies (Brown \& Hagoort, 1993). Nevertheless, in an attentional blink paradigm, where awareness of a stimulus can be prevented by having it follow an attention-catching target closely in time, Luck, Vogel, and Shapiro (1996) demonstrated N400s that were sensitive to the semantic qualities of words that could not be reported. On balance, the results regarding the processing nature of the $\mathrm{N} 400$ are mixed. On the basis of the literature, the prospects for finding $\mathrm{N} 400$ effects in a masked semantic priming paradigm seem mildly discouraging. However, an important aspect of the method has been changed in our study relative to the two earlier ones (Brown \& Hagoort, 1993; Münte \& Heinze, 1991). Whereas these authors, following the standard behavioral paradigm, used brief and masked primes and measured effects on the ensuing full-view targets, we reversed the sequence, using full-view primes to measure the effects on brief and masked targets. For reasons that are discussed later, we believe this arrangement to be more conducive to measurable psychophysiological effects.

\section{Verbal Report}

In the present context, a psychophysiological indicator of semantic processing offered several advantages. Because the ERP was recorded while the word was being processed, there was no time lag between the marginally perceptible word and the measurement of its semantic activation. Furthermore, the verbal report allowed us to conditionalize data analysis on the success or failure of identifying individual words. Thus, we expected to find an N400 difference between in-category and out-of-category words among the identified words. The crucial test of unconscious processing is whether the same difference can be found among unidentified words as well.

The verbal report measure is open to the criticism that it leaves the setting of the response criterion to the participant (Eriksen, 1960). When in doubt, a participant may withhold his or her response in spite of having had a partial perception. Two considerations help alleviate this concern. First, participants can be encouraged to guess when they are uncertain, and this was done in the present study. Second, a partial perception is of little help to the participant in performing the semantic task, because being aware of a few letters or letter fragments does not answer the question of whether the word belongs to the given category, and the partial perception is therefore useless in shaping the $\mathrm{N} 400$ response.

Still, the problem of potential underreporting of low-confidence responses is at the nexus of the present methodology. Therefore, it is worth considering some other methodological advances that have been proposed to clarify the relations between subjective and objective measures. The object is to detect an outcome for which genuinely unconscious processing has taken place and separate it from an outcome where some trials with conscious perception slip through the net and masquerade as unconscious processing.

\section{Qualitative Differences}

Following a suggestion by Dixon (1971), Cheesman and Merikle $(1985,1986)$ have proposed that qualitative differences between processing above and below the threshold can perform such a separation. An example from the literature is Marcel's (1980) finding that polysemous words activate all of their meanings when processed unconsciously but only the contextually appropriate meaning when processed consciously. Further, Cheesman and Merikle (1986) have shown that strategic expectancies play a part in conscious but not unconscious processing in the Stroop task. Additionally, Merikle and Reingold (1990) have demonstrated that words, in contrast to nonwords, give better than chance recognition after unconscious perception, whereas both words and nonwords are recognized after conscious perception. Thus, when differences in the type of processing can be demonstrated, underreported conscious processing becomes less likely as an explanation. In the present context, the locus of processing in the brain may serve this function.

In the localization of cognitive processing, hemispheric asymmetries form a conspicuous and extensively researched area. It is well known that language processing is normally lateralized to the left hemisphere, but it has also been found that elementary, receptive language processing can take place in the right hemisphere (Beeman \& Chiarello, 1998). For ERP amplitudes in the N400 time range, consistent asymmetries have been found over central and parietal areas, with the left side being more positive than the right (Kutas, Van Petten, \& Besson, 1988). Therefore, the degree of processing lateralization seems to be a characteristic worth examining that is potentially capable of distinguishing semantic word processing above threshold from processing below it.

This hypothesis needs some clarification. Brain ERPs are often shaped by partly overlapping components, with the blend being determined by task and stimulus characteristics. For the task we selected, ERPs in the crucial time span are typically dominated by a large, extended, positive-going complex that often accompanies cognitive processing. Superimposed on it rides the negative-going component called the $\mathrm{N} 400$, which is larger the more unexpected the stimulus is (i.e., the more difficult it is to assimilate into context). However, even with the most N400-provoking stimuli, the wave shape tends to be dominated by the positive complex. When we formulate hypotheses about localization, we need to distinguish between two related phenomena. First, there is the difference between in-category and out-of-category words, henceforth called the semantic priming effect. We expect this to have a central maximum, and possibly a slight right-over-left asymmetry, because this is the typical finding in the literature. The semantic priming effect is expected to be more pronounced for identified 
words, but the hypothesis of unconscious semantic processing predicts that it will be found for unidentified words as well, with the same localization and general characteristics, except possibly for size.

In contrast, the raw amplitudes in the same time band tend to be dominated by the positive complex for both in-category and outof-category words. It is in this primary, underived measure that we expect to find a difference between identified and unidentified words. For identified words, processing is expected to reach its characteristic hemispheric specialization, but for unidentified words, this may not be the case. When we refer to qualitative differences, it is this effect on raw amplitudes that we mean. The effect is operationalized as an interaction between word identification (averaged across in-category and out-of-category words) and lateral electrode site (left-center-right) over parietal areas.

In other words, the semantic priming effect reflects processing of the semantic relation between prime and target, and we expect it to be qualitatively similar between identified and unidentified words. Raw amplitudes reflect other, complementary aspects of the cognitive processing of the words, and we expect them to show different degrees of lateralization for different levels of conscious identification. This latter relationship, if it can be established, will serve to distinguish processing below subjective threshold from processing above it, in the manner demanded by Cheesman and Merikle's (1985, 1986) approach.

\section{The Regression Method}

The possibilities of distinguishing conscious from unconscious processing are not exhausted by the verbal report measure. In the present task, when the participants reported what word, if any, they had seen, they also made a binary choice concerning whether the word, identified or not, belonged to the given category. This choice variable can form the basis of a $d^{\prime}$ sensitivity measure, conditionalized on word identification. If an objective threshold is lower in terms of stimulus energy than a subjective threshold (Cheesman \& Merikle, 1985; 1986), then the binary-choice $d^{\prime}$ may exceed zero for unidentified words, which shows paradoxically that participants can guess at levels better than chance concerning the semantic qualities of words that they cannot report. Above-zero sensitivity for unidentified words can be interpreted in different ways: as a result of unreported conscious processing in a certain proportion of trials, as a result of participants' accessing the end result of unconscious semantic processing, or as a combination of both. In either case, the binary-choice variable can be used to provide a very strict test of the psychophysiological outcome. If the sensitivity of the $\mathrm{N} 400$ exceeds that of the semantic binary choice, then a strong argument has been built for unconscious processing, because the choice variable is an exhaustive measure of conscious semantic processing. Raising the expectations of psychophysiological sensitivity to this high level ensured that we would err only on the side of caution in using our psychophysiological data as a basis for concluding that unconscious semantic processing had occurred.

A method for comparing the sensitivity of different measures of semantic processing has recently been developed (Draine \& Greenwald, 1998; Greenwald et al., 1996; Greenwald, Klinger, \& Schuh, 1995). It is a regression method that takes advantage of the interindividual variation to regress the measure of unconscious processing (the indirect measure) on the measure of conscious processing (the direct measure). If the regression function has an intercept reliably above zero, then it has been demonstrated that the indirect measure is the more sensitive of the two and, by extension, that unconscious processing takes place when conscious processing does not. Such an outcome has been demonstrated repeatedly by Greenwald and his colleagues. In the present context, regressing the psychophysiological measure on the binarychoice variable served the same function, and a reliably positive intercept bolstered a potential case for unconscious processing.

\section{Objective Threshold}

Up to this point, we have presented a psychophysiological indirect measure and two varieties of direct measures: a verbal report and a semantic binary choice. The former instantiates the more lenient subjective threshold by being an exclusive, but possibly not exhaustive, measure of conscious processing. The latter represents an objective threshold and provides a measure that is exhaustive to the point of being nonexclusive. However, objective thresholds can be instantiated in different ways. A further possibility is a forced-choice identification of the displayed word among a given number of alternatives. In Experiment 2, a twoalternative, forced-choice procedure was applied, and in Experiment 3 a six-alternative, forced-choice was used. Using a forcedchoice test removed the problem of response bias that impugns the oral identification measure.

Psychophysiologically, the total number of trials for each experimental participant was separated into four cells, each of which formed an averaged ERP. The basis of this $2 \times 2$ partition was category membership-nonmembership of the word and successfailure in word identification. Identification by verbal report was of considerable intrinsic interest because of its face validity, and it was used as a basis for averaging in all three experiments. In addition, we used an objective-threshold measure as an averaging basis. For this purpose, two-choice variables, such as the semantic binary-choice (Experiments 1-3) or the two-alternative, forcedchoice (Experiment 2) were less well suited, because the high prior probability of guessing correctly would have overestimated conscious processing and underestimated unconscious processing. To avoid this, a six-alternative, forced-choice response in Experiment 3 formed the basis of trial averaging. Thus, whatever semantically based differences we may find among verbally unidentified words will gain in strength if they also appear among words unidentified by the stricter standard of the forced-choice test.

\section{Overview of Experiments}

The approach used in our experiments was one of converging evidence. The basic design was simple: We looked for semantically based differences among unidentified words. In the definition of what it means to be unidentified, we used a multilayered approach. Verbal report identification (i.e., a subjective threshold) was the foundation, because of its obvious face validity. To counter objections leveled at the concept of a subjective threshold, we investigated possible differences in the localization of processing above and below threshold, reasoning that such differences may vindicate the definition.

Objective measures of identification were applied in two ways. The straightforward approach was to use success or failure in the 
selection among alternatives as the definition of identification. This was done in the final experiment. The alternative was to apply the regression method of Greenwald et al. (1996) to the set of orally unidentified trials. With this method, by first weeding out trials where identification obviously had taken place, we restricted analysis to cases where the oral identification standard may have possibly misled us. By scrutinizing this set with the aid of an objective, binary-choice test, we could determine whether objective choice performance completely explained the semantic ERP effect. The issue to be tested was whether the ERP effect was still in force (intercept $>0$ ) when choice performance had come to the end of its tether $\left(d^{\prime}=0\right)$. The regression approach was used in all our experiments.

As a priming experiment, the present study reversed a few features of the customary design in the literature on subliminal semantic activation. Normally, a marginally perceptible word is displayed as a prime, and its effect on processing of the ensuing target is assessed. Here, the prime was clearly visible and the target marginally perceptible, allowing us to measure both semantic effect and detectability on the same word. The absence of a time lag relieved us of some potential hazards in the standard design, such as decay of activation over time and interference between memory encoding of the prime (for report) and processing of the target (Fischler \& Goodman, 1978). The latter feature-report of individual items-is not often included in the standard design (but see Hirshman \& Durante, 1992). For present purposes, it allowed us to conditionalize psychophysiological averaging on identification data, thus relieving us of reliance on identification performance measured in a separate block or session.

We used a relatively narrow span of display durations, letting performance do the sorting into sub- and suprathreshold conditions. Avoiding the more frequent approach of clearly separated display durations allowed us to minimize superficial differences in perceptual processing between the two conditions, because any such differences could have been confounded with the identifiedunidentified dichotomy in the ERPs. Also, it has been found that mixing long and short exposures reduces priming for the shortexposure trials (Smith, Besner, \& Miyoshi, 1994; Stolz \& Besner, 1997). ${ }^{1}$

\section{Experiment 1}

Experiment 1 explored the basic method in a relatively small sample of participants. A limited set of electrodes was used, covering the conventional midline sites while giving some indication of lateralized processing in parietal areas. The stimulus material consisted of a small set of target categories. The category name that served as a basis for the category judgment was the same throughout a block that consisted of 30-66 trials.

\section{Method}

\section{Participants}

Thirteen students at the University of Lund ( 7 women, 6 men) participated and were paid $200 \mathrm{kr}(\$ 24)$. Ages ranged from 20 to 31 years ( $M=24.3$ years). All participants described themselves as right handed.

\section{Procedure}

Stimuli were displayed on a Macintosh II vi computer. After a practice session with 26 trials, the experimental session, comprising 340 trials, ensued. The session was divided into seven blocks, each of which had a different target category (e.g., "clothes"). The category name was shown in a dialog window at the outset of the block, and the participant was instructed to keep it in mind for the rest of the block. Each trial started with a fixation cross shown for $1 \mathrm{~s}$ in the word display window, which was located at the center of the screen. A word was briefly displayed, followed by a mask-a wicker-like pattern that filled the display window. The mask remained until the participant gave a categorization response with the computer mouse button (a single click or a double click). When the mask had been cleared, an icon prompted the participant to give an oral response. The experimenter keyed in an evaluation of the response (correct, no response, wrong guess). Feedback was then given on screen, showing the target category, the displayed word, and whether the participant had categorized it correctly or not.

The critical masked stimulus words were shown under conditions that were meant to offer some variability around identification threshold for most participants. For this purpose, six display levels were used, produced by crossing two durations ( 33 and $50 \mathrm{~ms}$ ) with three contrast levels (39\%, $71 \%$, and $88 \%$ ). (In computer displays, variation in duration is constrained to be multiples of the screen refresh cycle. Software-controlled contrast can produce additional variation.)

Our monitor was set to display 16 shades of gray, numbered from 0 to 15 . A default text display using black text on a white background would give a contrast value of $97 \%$, which was obtained by inserting measured luminances $3.4 \mathrm{~cd} / \mathrm{m}^{2}$ (Shade 0 ) and $115 \mathrm{~cd} / \mathrm{m}^{2}$ (Shade 15) into the following formula: Contrast $=[($ Background - Foreground $) /$ Background]. Contrast levels used in this experiment were as follows:
a. $39 \%$. Text, Shade $6\left(34 \mathrm{~cd} / \mathrm{m}^{2}\right)$ and background, Shade 9 (56 $\left.\mathrm{cd} / \mathrm{m}^{2}\right)$.
b. $71 \%$. Text, Shade $4\left(21 \mathrm{~cd} / \mathrm{m}^{2}\right)$ and background, Shade $11(73$ $\left.\mathrm{cd} / \mathrm{m}^{2}\right)$.
c. $88 \%$. Text, Shade $2\left(11 \mathrm{~cd} / \mathrm{m}^{2}\right)$ and background, Shade $13(94$ $\left.\mathrm{cd} / \mathrm{m}^{2}\right)$.

\section{Recording}

A NeuroScan system was used, here and in the following experiments, for data acquisition and off-line postprocessing. Ag-electrodes were applied to six scalp positions: $\mathrm{Fz}, \mathrm{Cz}, \mathrm{P3}, \mathrm{Pz}, \mathrm{P} 4$, and $\mathrm{Oz}$ (see Figure 1), using linked earlobes as reference. Vertical eye movements were monitored by electrodes above and below the left eye and horizontal eye movements by two electrodes outside the outer canthi of the eyes. Analog filters passed a band of frequencies from 0.7 to $45.0 \mathrm{~Hz}$ (3-dB attenuation points). Subsequent off-line signal processing included digital low-pass filtering with $20 \mathrm{~Hz}$ as cut-off (slope $=12 \mathrm{~dB} /$ octave). The EEG was sampled at $250 \mathrm{~Hz}$, covering an epoch from $300 \mathrm{~ms}$ prestimulus to $1,500 \mathrm{~ms}$ poststimulus and saved to disk for off-line analysis. Corrections for EOG activity were made using NeuroScan's software. Trials containing movement artifacts were rejected by using a combination of preset voltage criteria and visual inspection. Baseline correction was performed by subtracting the mean of the prestimulus epoch $(300 \mathrm{~ms})$ from each data point.

The computer that presented the stimuli tagged each trial with a characteristic trigger byte that was saved with the EEG data. In postprocessing, the behavioral response data (indicating success or failure in word identification) were combined with the EEG data to allow averaging of all trials into four cells: identified in-category words, identified out-of-category words, unidentified in-category words, and unidentified out-of-category words.

\footnotetext{
${ }^{1}$ In an early, small-scale (11 participants) study, we used two widely separated display durations, and the effects found in the experiments reported here were not obtained.
} 


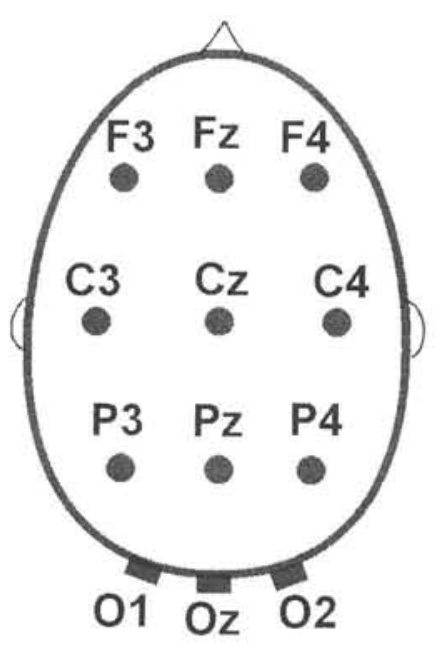

Figure 1. Schematic diagram of electrode positions. Experiment 1 used the midline sites $(\mathrm{Fz}, \mathrm{Cz}, \mathrm{Pz}$, and $\mathrm{Oz}$ ) and the parietal sites $\mathrm{P} 3$ (left) and $\mathrm{P} 4$ (right).

\section{Stimulus Material}

A total of 340 stimulus words were used. Of these, 170 were targetcategory exemplars, belonging to seven categories (birds, fruit and vegetables, four-legged animals, clothes, kitchen utensils, body parts, and furniture). The other 170 were distractors and were also common nouns. The two types of words were similar in physical appearance when compared for word length, word width in pixels, and total number of pixels occupied by word. The words (Swedish, with English translations) along with various item statistics can be found on the Internet at http://www. psychology.lu.se/Personal/Stenberg/SemanticERPs/. Other aspects of the word items are discussed in the General Discussion.

A list was produced by randomly mixing the category words with an equal number of distractors within each category block. Exposure durations and contrast levels were randomly assigned to the stimuli. The same list was used for all participants.

\section{Results}

\section{Word Identification}

The overall rate of word identification was $69 \%$. As expected, the proportion of correct identification varied with display conditions as an increasing function of duration and contrast. (Identification data for the six combinations of duration and contrast were as follows: [duration $=33 \mathrm{~ms}$, contrast $=39 \%$ ] $-4 \%$ identification; [33 ms, 71\%]—69\%; [33 ms, $88 \%$ ] - $86 \%$; [ $50 \mathrm{~ms}, 39 \%$ ]$59 \%$; [50 ms, $71 \%]-97 \%$; [50 ms, $88 \%]-98 \%$.)

Responses in trials without successful identification $(31 \%)$ were distributed as follows: "Don't know" $=22 \%$, wrong guesses $=8 \%$, close guesses (more than 1 syllable in common with the correct word; these trials were discounted from ERP averaging) $=2 \%$.

There was a priming effect on word identification, such that in-category words were identified more easily $(72 \%)$ than out-ofcategory words $(65 \%)$. (The mean number of trials [range within brackets] included in the ERP averages were: identified incategory [IdIn] $=119$ [97-140], IdOut $=108$ [78-127], UnidIn $=44[27-67]$, and UnidOut $=53[37-81]$.

\section{Binary Choice}

The signal-detection measure of sensitivity, $d^{\prime}$, was computed for the forced binary categorization of unidentified words (categorization of identified words was, as expected, virtually perfect). The mean $d^{\prime}$ of .07 (95\% confidence interval from -0.22 to 0.36 ) was not significantly different from zero, $t(12)=0.53$. Thus, participants were not able to categorize unidentified (by verbal report) words better than chance.

\section{ERPs}

An interval was defined in which the $\mathrm{N} 400$ effect was expected. This interval extended from 400 to $600 \mathrm{~ms}$, and average amplitude in this time window was the main dependent variable in the analysis of ERPs. Grand average wave forms are shown in Figure $2 \mathrm{~A}$ and Figure 2B.

A three-way analysis of variance (ANOVA) was computed, using mean $400-600$-ms amplitude at the three midline sites Fz, $\mathrm{Cz}$, and $\mathrm{Pz}$ as dependent variables. The variables were word identification (two levels), category membership (two levels), and electrode site (three levels).

The effect of primary importance was the category main effect, which proved reliable, $F(1,12)=28.87 ; M S E=2.73$. (Here and in the following, $p<.05$ was adopted as the level of significance.) As shown in the figures, there was a difference in the critical interval between wave forms evoked by category members and those evoked by category nonmembers, with the latter being more negative-going, as could be expected from an N400 effect. Marginal means showed that the average difference was $1.42 \mu \mathrm{V}$. Further elucidation of the category effect demanded separate analyses for identified and unidentified words.

Among identified words, the category effect was clearly reliable, $F(1,12)=19.91, M S E=2.07, \eta^{2}=0.62$. Mean amplitude was reduced by category deviations from 3.21 to $1.75 \mu \mathrm{V}$. However, the critical test concerned unidentified words. For these, the effect was also reliable, $F(1,12)=7.50 ; M S E=5.04 ; \eta^{2}=0.39$. Paralleling the results for identified words, mean amplitude in the interval was less positive $(3.26 \mu \mathrm{V})$ for out-of-category than for in-category words $(4.65 \mu \mathrm{V})$.

\section{Qualitative Differences}

To determine whether the processing of identified words was localized differently from that of unidentified words, mean amplitudes from the critical interval were analyzed for the three parietal electrodes $\mathrm{P} 3$ (left), $\mathrm{Pz}$ (center), and P4 (right). The analysis comprised three variables: site (three levels), identification (two levels), and category (two levels). The main point of the analysis was to determine whether the left-right gradient differed between identified and unidentified words. In ANOVA terms, it translates into an interaction between the Identification factor and the linear contrast across the site factor.

The ANOVA showed, first, that the Category factor was reliable for these electrode locations as well, $F(1,12)=24.21$, 
$M S E=3.70, \eta^{2}=0.67$. There were simple main effects of category among both identified, $F(1,12)=21.42, M S E=2.82$, $\eta^{2}=0.64$, and unidentified words, $F(1,12)=4.76, M S E=6.62$, $\eta^{2}=0.28$.

The crucial interaction between identification and the linear site contrast was reliable, $F(1,12)=17.58, M S E=0.29 .^{2}$ Decomposition showed a linear trend for identified words, $F(1,12)=6.50$, $M S E=1.54$ (left > center > right; see Figure 3). There was no such trend among the unidentified words, $F(1,12)=0.00$; $M S E=0.61$.

Thus, referring back to our earlier formulation of two hypotheses, there were qualitative differences in the lateralization of raw amplitudes. It is a different question whether the semantic priming effect changed its scalp distribution. This question was first approached by examining the Category $\times$ Identification $\times$ Site (linear trend) interaction, which was not reliable, $F(1,12)=3.61$; $M S E=0.12$. Taking a more general view, another test was performed, in which all recorded electrode sites took part. This test lets all possible differences in scalp distribution of the $\mathrm{N} 400$ effect emerge, without prejudging the issue where they are to be found. Differences between in-category and out-of-category words were computed in the 400-600-ms interval, normalized by McCarthy and Wood's (1985) length-of-vector method, and entered into a 2 (identification) $\times 6$ (electrode sites) ANOVA. The crucial effect concerned the Identification $\times$ Site interaction. It was not significant: $F(5,60)=1.33, \epsilon=0.52 ; M S E=0.09$.

\section{Regression Test}

To test whether the $\mathrm{N} 400$ effect was more sensitive to category membership in unidentified words than was the binary-choice $d^{\prime}$, a regression analysis was performed. A derived variable was constructed to gauge the $\mathrm{N} 400$ effect, namely, the difference between in-category and out-of-category amplitudes for the $\mathrm{Cz}$ electrode. Figure 4 shows this variable regressed on the $d^{\prime}$ variable.

It is critical that the intercept was 1.60 , which was reliably different from zero, $t(11)=2.63$. The slope, 0.52 , did not deviate significantly from zero, $t(11)=0.40$. A similar test, performed on amplitude values from the $\mathrm{Pz}$ electrode, showed almost identical results: A significant intercept $=1.60, t(11)=2.41$, and a nonsignificant slope $=0.37, t(11)=0.26$.

\section{Discussion}

Experiment 1, although small in scale and somewhat exploratory in nature, showed the hypothesized effects of semantic processing separate from subjective identification. There was a difference in brain responses between in-category and out-of-category words, and this difference was found in the time interval expected to show $\mathrm{N} 400$ effects. It is crucial that the difference was found for unidentified words as well as for identified ones. Admittedly, the wave shape assumed the characteristic peaked shape that is typical of visually defined ERP components only for identified words, not for unidentified ones. It is, however, not unusual for an ERP component to assume the form of a superimposed wave, rather than a visually defined peak. Furthermore, the relatively weak and probabilistic process that can be expected in unconscious semantic processing is not likely to assume the fixed and constant relation to stimulus display needed for the shaping of a well-defined wave crest. The localization of the effect, with a maximum over central and parietal areas, was compatible in general terms with what is known about the N400, although judgment on this score will have to await the use of a larger set of recording sites.

The examination of possible qualitative differences in the processing of identified and unidentified words revealed a left-right asymmetry for identified words, but not for unidentified ones. Keeping in mind the complexities involved in ascribing specific brain localizations to voltage patterns obtained on the scalp, further discussion of the localization issue are deferred to a later section. Suffice it here to note that different neural populations seemed to be involved in processing identified and unidentified words, and this fact vindicates the usefulness of the subjective identification criterion.

Furthermore, the objective test, a yes-no categorization of the unidentified words, showed sensitivity to be indistinguishable from zero. Additionally, the regression procedure indicated that sensitivity at exactly zero would be associated with an ERP effect that significantly exceeded zero. This test suggests that the use of a subjective criterion for identification is not essential for establishing ERP semantic effects among unidentified words-an objective threshold would show the same result. A further discussion of issues involved in the use of the regression approach appears in a later section.

A number of aspects of the methodology of this first experiment can be improved. First, there were few participants and, consequently, low power to reject null hypotheses. This means that null results, such as the chance-level, binary-choice $d^{\prime}$ and the null slope in the regression test, have to be interpreted with caution. Second, the filtering characteristics of the amplifiers were not ideal for capturing the relatively slow electrophysiological phenomena we were dealing with, and amplitudes of some of the slow potentials may have been curtailed as a result. Third, stimulus display conditions were not well suited to achieving the desired balance between identified and unidentified words, which would ideally result in $50 \%$ of each to guarantee an efficient use of the experimental session. Fourth, a larger array of electrodes was needed to permit conclusions on the spatial distribution of processing. Fifth, trials were not primed with the target category immediately before stimulus displays, a fact that may be responsible for the relatively weak N400 effect. A closer temporal contiguity between category primes and stimulus words may improve the strength of the N400generating process, and a slightly different procedure was therefore tried in the next experiment.

\section{Experiment 2}

Experiment 2 replicated the basic design of the first while addressing some of the concerns raised by its predecessor. There was a larger number of participants tested with recording equipment, with a larger array of electrodes and more suitable amplifiers. The stimulus presentation procedure was altered to introduce a category prime before each trial, and the presentation of masked words was refashioned to use a smaller set of display levels, the

\footnotetext{
${ }^{2}$ Following the recommendations of McCarthy and Wood (1985, p. 206), this interaction effect was also examined in an ANOVA where amplitude values were rescaled by vector length. It remained reliable, $F(1$, 12) $=15.46, M S E=0.005$.
} 
A
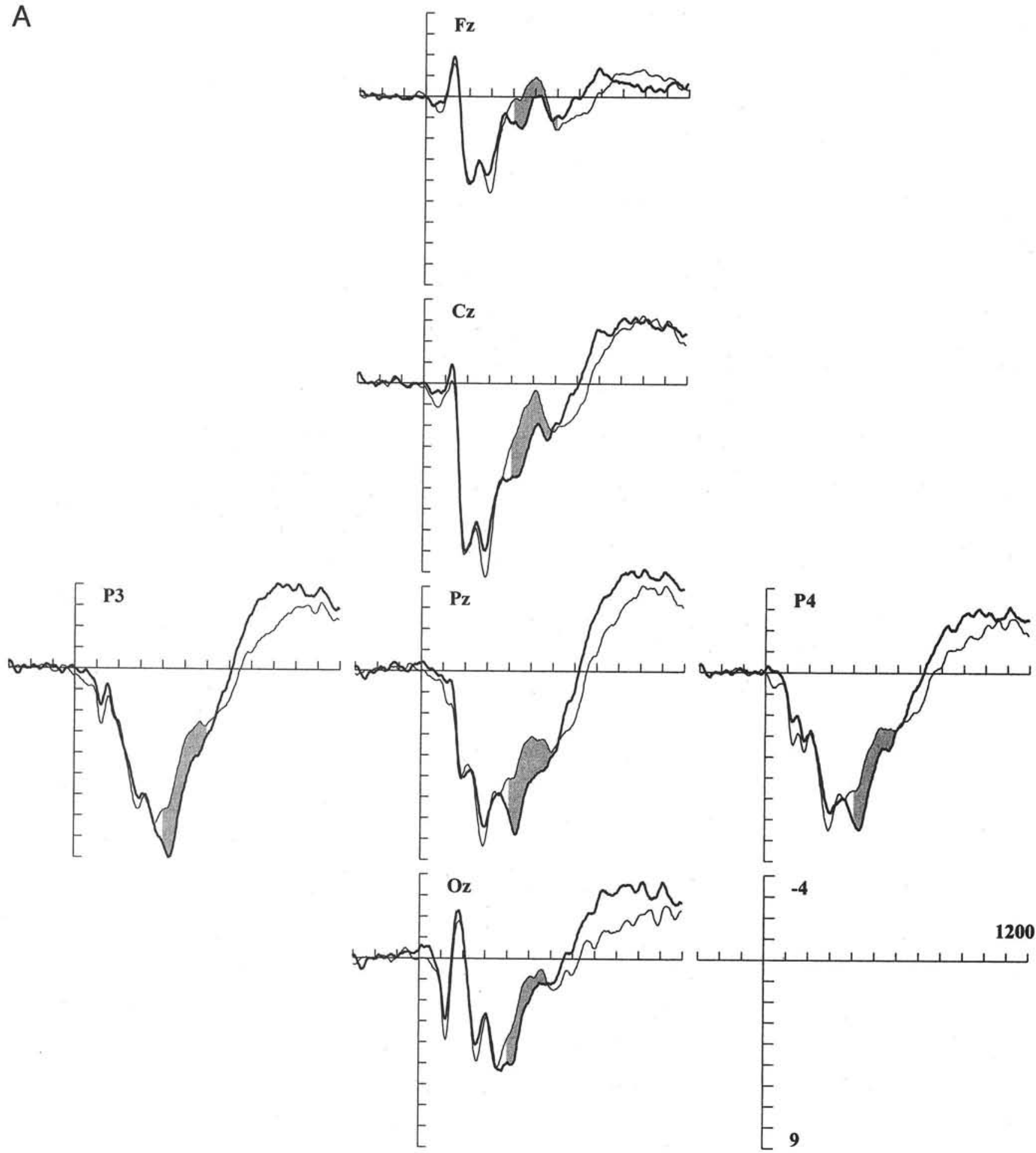

Figure 2A. Experiment 1: Event-related potential (ERP) wave forms for in-category (bold line) and out-ofcategory (thin line) words, plotted for identified words. The difference between in-category and out-of-category words is shaded in the critical time interval $(400-600 \mathrm{~ms})$. 
A
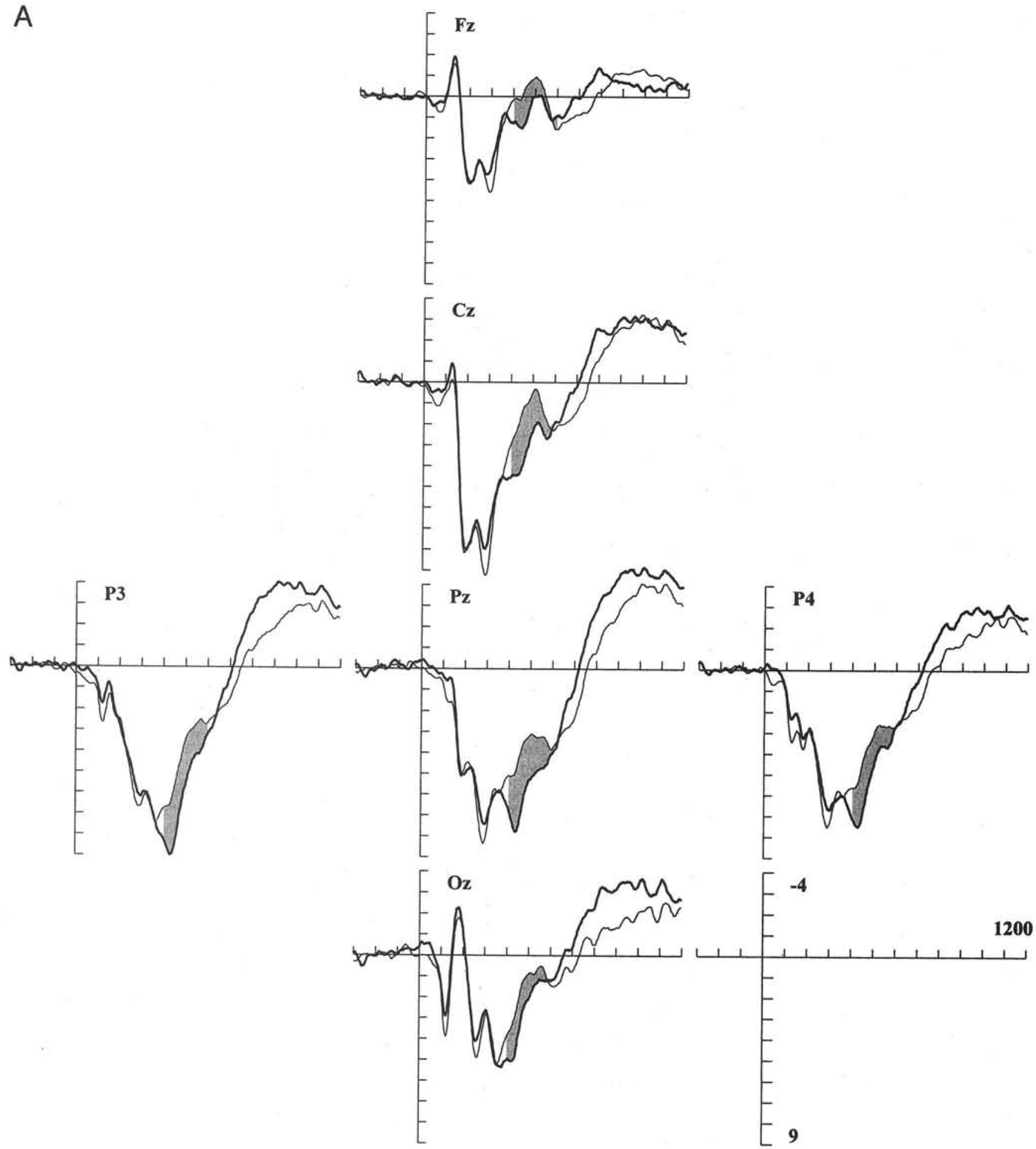

Figure 2B. Experiment 1: Event-related potential (ERP) wave forms for in-category (bold line) and out-ofcategory (thin line) words, plotted for unidentified words. The difference between in-category and out-ofcategory words is shaded in the critical time interval $(400-600 \mathrm{~ms})$. 


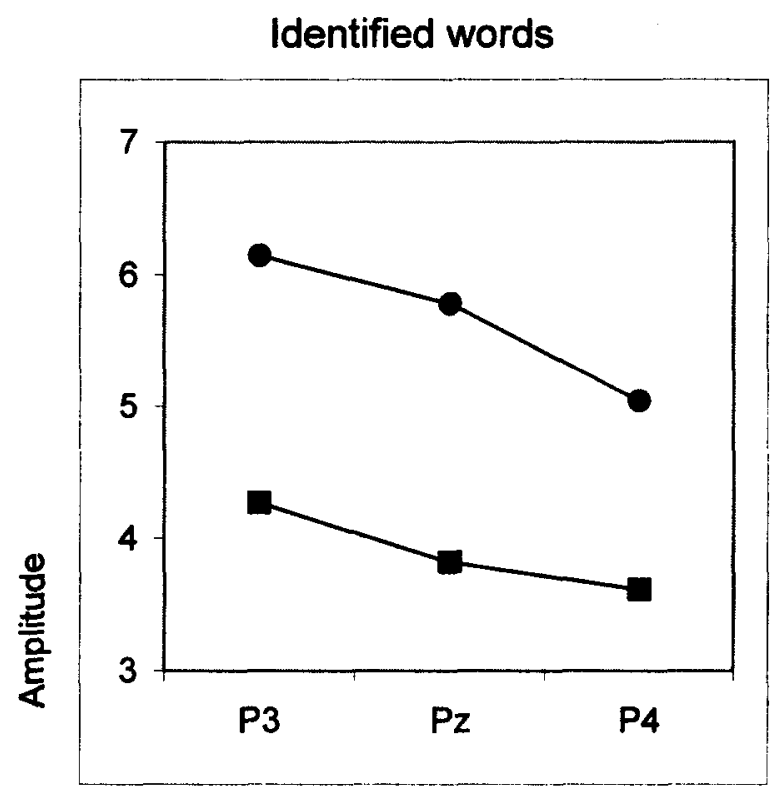

\section{Unidentified words}

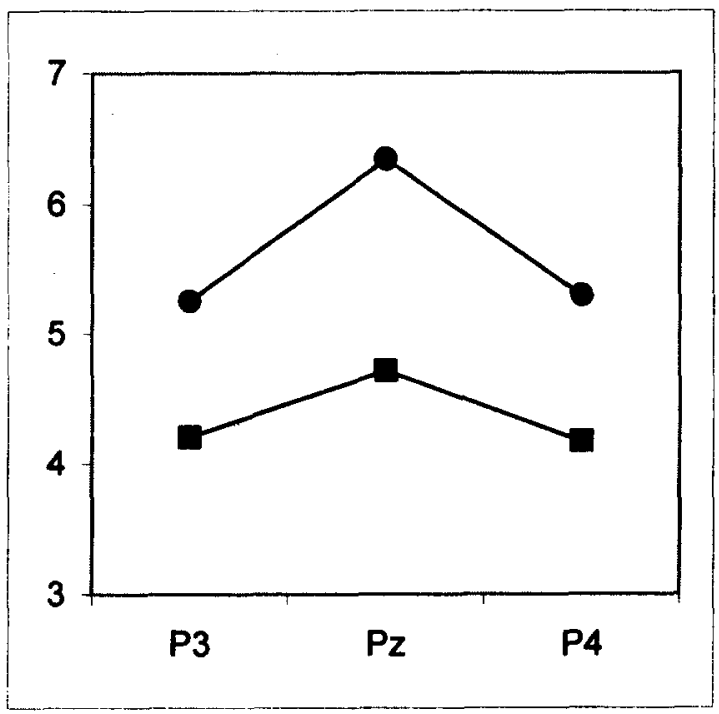

Identified words

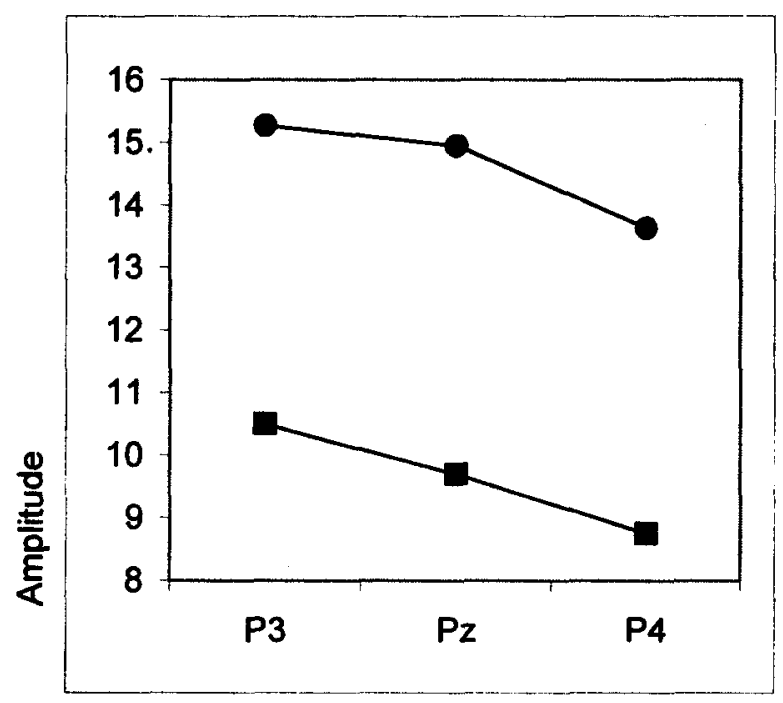

Electrode site

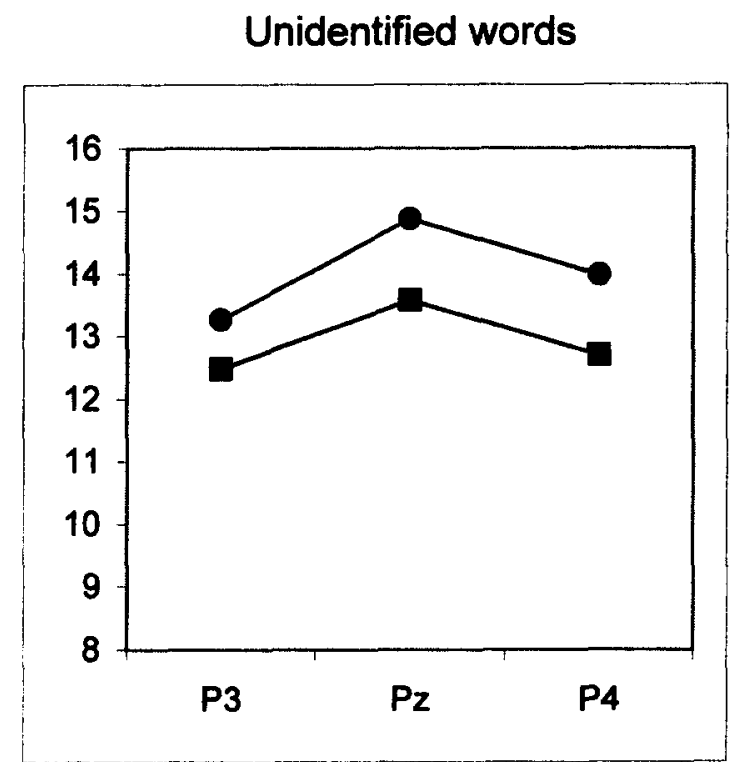

Electrode site

Figure 3. Mean amplitudes in the 400-600-ms band at three parietal sites for Experiment 1 (top) and Experiment 2 (bottom). Round markers show in-category words, square markers show out-of-category words. Both experiments show category effects for unidentified as well as for identified words but also a left-over-right asymmetry for identified but not for unidentified words.

admixture of which was titrated to fit each participant optimally. Furthermore, an additional test of word identification was introduced, in that a two-altemative, forced-choice procedure was given in each trial. Thus, apart from the electrophysiological variables, participants gave three behavioral responses to each stimulus word, in turn: yes-no categorization, oral identification, and a forced choice between two word alternatives.

\section{Method}

\section{Participants}

Thirty-four students at the University of Lund were tested and paid 200 kr (\$24) for their participation. Three participants were excluded because of equipment malfunction and 1 participant because he reported being unable to attend to the masked stimuli. Of the remaining 30 participants, 17 


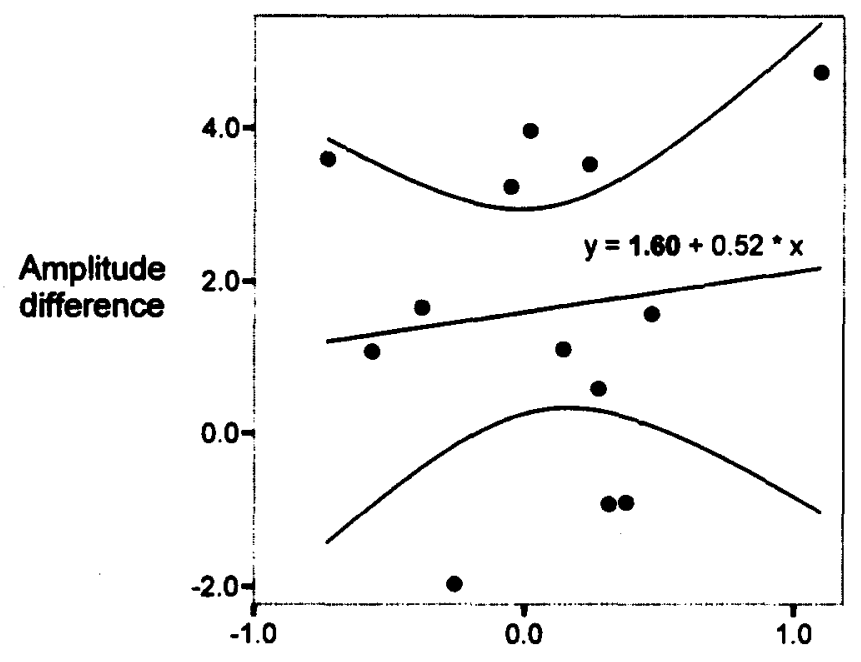

d'

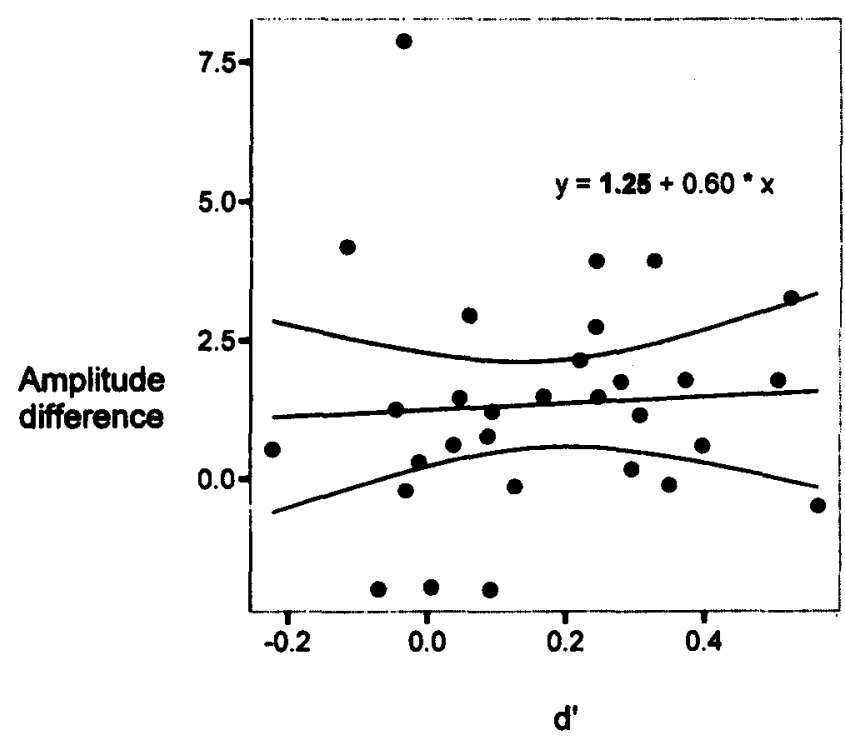

Figure 4. Scatterplot with regression line and $95 \%$ confidence interval (curved lines) for Experiment 1 (top) and Experiment 2 (bottom). The ordinate shows the amplitude difference between in-category and out-ofcategory words at the $\mathrm{Cz}$ site for unidentified trials. The abscissa shows $d^{\prime}$ in semantic binary choice for unidentified words. Boldface type is used in regression equations for intercepts or slopes that are significantly different from zero.

were men and 13 were women. Mean age was 23.2 years (range $=20-30$ years).

\section{Recording}

Ag electrodes were applied to 11 scalp positions: F3, Fz, F4, C3, Cz, C4, P3, Pz, P4, O1, and $\mathrm{O} 2$ (see Figure 1), using linked earlobes as reference.
Vertical eye movements were monitored by electrodes above and below the left eye and horizontal eye movements by two electrodes outside the outer canthi of the eyes. Analog filters passed a band of frequencies from 0.1 to $50.0 \mathrm{~Hz}$. Subsequent off-line signal processing included digital filtering, which produced a band pass of $0.1-20.0 \mathrm{~Hz}$, with a 24-dB/octave roll-off. The EEG was sampled at $250 \mathrm{~Hz}$, covering an epoch from $300 \mathrm{~ms}$ prestimulus to $1,200 \mathrm{~ms}$ poststimulus, and saved to disk for off-line analysis. Trials containing artifacts caused by muscle movements or blinking at the time of stimulus delivery were rejected. Corrections for remaining EOG activity were made using NeuroScan's software. Baseline correction was performed by subtracting the mean of the prestimulus epoch (300 ms) from each data point.

\section{Stimulus Material}

A number (308) of word triplets were constructed, in which one word was a category label (e.g., "flower"), another was an instance of that category (e.g., "tulip"), and the third was an unrelated word (e.g., "virus"). The category label was used to cue the task for the participant ("Does the masked word you are about to see belong to this category?"). In half of the trials the category instance was shown, and in the other half the unrelated word was shown; the selection of one or the other was made on a pseudo-random basis by the program at run time.

In all, 90 category labels were used, such as flower, fruit, building, country, metal, musical instrument, month, profession, fuel, mammal, vehicle, and fish. Most words were nouns, and some were proper names (e.g., names of countries). The words, their translations, and further statistics are shown on the Web site mentioned in Experiment 1. All words were shown with the first letter capitalized, so that proper names could be shown with their normal spelling without being singled out orthographically. The two types of words were matched for length and size. From the pool of 308 triplets, the experiment program made a random selection of 6 for the practice block and 240 others for the actual trials. The selection was new for each participant, as was the ordering of the stimuli.

\section{Procedure}

The entire testing session, excluding electrode application, took about 90 min. Short breaks (including on-screen feedback about performance) were inserted after each of eight blocks, and a longer break (about $5 \mathrm{~min}$ ) after the fourth block.

Stimuli were displayed on a 13-in. (33.02 cm) Apple monitor, driven by a Macintosh II $\mathrm{fx}$ computer under the control of a Pascal program. The screen was set to a middle-gray shade, except for a white rectangular window at the center of the screen, approximately $60 \mathrm{~mm} \times 15 \mathrm{~mm}$, in which the stimuli were shown. All word stimuli were shown using black, 24-point characters from the Palatino font on a white background. The masked stimuli were both preceded and followed by masks, which were different from each other and varied from trial to trial, to increase the difficulty of reading the words. The masks consisted of strings of 12 Chinese ideographs. They were constructed by random selection with replacement from a set of 28 characters in a True Type Chinese font and displayed in a 24-point size.

There were three exposure durations for the masked words: one, two, or three multiples of the vertical blanking interrupt frequency of $60 \mathrm{~Hz}$ (i.e., 17, 33, and $50 \mathrm{~ms}$ ). Initially, one third of the stimuli was assigned to each of these three exposure durations. To achieve the desired rate of $50 \%$ word identification, an adaptive algorithm went into operation, which adjusted the difficulty of the task to the performance of the participant. The algorithm worked on blocks of 30 stimuli. After the completion of one such block, the program determined the discrepancy between the desired identification rate and the actual and reassigned trials in the upcoming block from the easiest to the hardest exposure duration (or vice versa) to counteract the imbalance. Thus, because most of our participants succeeded in 
reading somewhat more than half of the masked words, they met an increasing proportion of hard $(17 \mathrm{~ms})$ trials during the experiment. They saw a corresponding decrease in easy $(50 \mathrm{~ms})$ trials, whereas the proportion of intermediate ( $33 \mathrm{~ms}$ ) trials was held constant throughout.

A trial consisted of three main events, interwoven with the responses made by the participant: the display of the category prime, the display of the masked word, and the display of the two unmasked word alternatives. The participant's responses were made by selections in dialog windows. First, the category label (e.g., "fish") was shown in a dialog window, and the participant acknowledged the information by clicking an "OK" button. The dialog window disappeared and revealed a warning cross in the word-display rectangle. After $500 \mathrm{~ms}$, the warning was succeeded by a premask of Chinese characters, shown for $1,000 \mathrm{~ms}$. The display word immediately followed, shown for one of the three display durations $(17,33$, or $50 \mathrm{~ms}$ ). A postmask replaced it, remaining for $1,000 \mathrm{~ms}$. Then, a dialog window presented the two response alternatives "Yes, the word belonged to the category" and "No, it did not", and a selection had to be made before the dialog window disappeared. It was immediately followed by another dialog, presenting the three alternatives "I saw the word", "I am guessing", and "I don't know". Icons (and previous instructions) indicated that the first 2 alternatives were to be accompanied by an oral response. When the dialog window had been dismissed, a new warning cross $(500 \mathrm{~ms})$ in the word-display rectangle ushered in the first of two semantically unrelated word alternatives, shown for $1,000 \mathrm{~ms}$ and followed by a 200 -ms blank. (ERPs were recorded when the word alternatives were shown, but they are not reported here.) The second word alternative was likewise preceded by a $500-\mathrm{ms}$ warning cross, shown for $1,000 \mathrm{~ms}$, and followed by a $200 \mathrm{~ms}$ blank. A dialog with the text "The displayed word was:" then forced a choice between buttons marked "Alternative \#1" and "Alternative \#2." Finally, a dialog with the text "The word I chose is the one I said aloud" gave the alternatives yes and no. The latter choice was used to mark the trials as successful in word identification or not.

\section{Results}

\section{Word Identification}

The adaptive testing procedure aimed at stabilizing participants' word-identification rates at or near $50 \%$, and it was successful in this regard. On average, $53 \%$ of the words were identified, with a range of $48 \%$ to $57 \%$ over participants. (Stimulus classes on which ERP averages were formed averaged as follows: 50 [Identified-1 Category+], 63 [Id-/Cat-], 70 [Id +/Cat + ], and 57 [Id +/Cat-] stimuli per condition. The minimum number of stimuli for any participant was 44 [these figures are given before artifact exclusion].)

The adaptive procedure resulted in $41 \%$ of the words being shown at the shortest duration, $33 \%$ at the intermediate, and $26 \%$ at the longest. Probabilities of identifying words at the three durations were as follows: $.09, .77$, and .93 . The probability of being right after expressing confidence about word identification was .97, whereas it was .62 after stating that the word was a guess. The abstention ("don't know") response was used in $37 \%$ of the trials and accounted for $79 \%$ of unsuccessful identification trials.

As expected from previous research, a semantic priming effect arose from the category cue, such that the probability of identifying a word that belonged to the cued category was .58 versus .48 for a miscued word. This overall priming effect $(0.10)$ was greatest at the intermediate level $(0.20)$ and smaller at the difficult $(0.04)$ and the easy levels $(0.08)$.

\section{Binary Categorization Performance}

Signal-detection sensitivity was computed for the binary choice of whether the word belonged to the cued category or not. This computation was performed for unidentified words only (performance for identified words was, as expected, virtually perfect). Average $d^{\prime}$ was 0.169 , with a $95 \%$ confidence interval from 0.094 to 0.243 . The mean was significantly different from zero, $t(29)=4.62$.

\section{Two-Alternative, Forced-Choice Performance}

Sensitivity $\left(d^{\prime}\right)$ was computed for unidentified words in the two-alternative forced choice of the recognition task. Average $d^{\prime}$

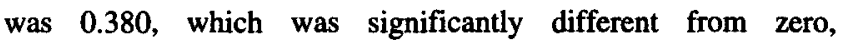
$t(29)=7.06$. It was also reliably larger than $d^{\prime}$ in the semantic categorization, $t(29)=4.03$. The two sensitivity measures were moderately correlated across participants $(r=.38)$.

\section{ERPs}

Grand average ERP curves are shown in Figure 5A and Figure 5B. An ANOVA was computed using average amplitudes for the three midline electrodes $\mathrm{Fz}, \mathrm{Cz}$, and $\mathrm{Pz}$ in the critical time interval as dependent variables. The ANOVA had three variables: word identification ( 2 levels), category membership ( 2 levels), and electrode site (3 levels).

Of primary interest is the category main effect, which was reliable, $F(1,29)=64.44, M S E=11.52$, and the Category $\times$ Identification interaction, which was also reliable, $F(1$, 29) $=62.23, M S E=4.37$. As can be seen in the figure, a large N400 component was invoked by out-of-category words, if they were identified. The N400 component had a centro-parietal maximum, and it reduced mean amplitude in the interval from 10.0 to $5.4 \mu \mathrm{V}$. Decomposing the interaction into simple main effects showed this effect to be highly reliable for identified words, $F(1$, 29) $=88.39, M S E=10.81, \eta^{2}=0.75$.

The crucial test, however, concerned unidentified words. The simple main effect of category on unidentified words proved also to be reliable, $F(1,29)=11.42, M S E=5.07, \eta^{2}=0.28$. As can be seen in the figure, the $\mathrm{N} 400$ effect was small but consistent, reducing mean amplitude from 10.6 to $9.5 \mu \mathrm{V}$ between the two category conditions. In amplitude terms, it amounted to $25 \%$ of the corresponding effect for identified words, and in statistical effect size terms (variance explained), $38 \%$.

\section{Qualitative Differences}

The processing of identified words was markedly asymmetrical, with larger (positive) amplitudes over left parietal sites than over corresponding areas in the right hemisphere. The processing of unidentified words was, however, largely symmetrical. This difference is also apparent in Figure 3, which shows mean amplitudes in the critical interval for the three parietal electrodes. 
An ANOVA was performed on mean amplitudes in the $400-$ 600 interval from the three parietal electrodes. In concordance with the midline test, there were effects of category, $F(1,29)=62.76$, $M S E=13.34$; of Category $\times$ Identification, $F(1,29)=71.62$, $M S E=4.66$; and there were simple main effects of category among identified words, $F(1,29)=81.61, M S E=13.65$, as well as among unidentified words, $F(1,29)=13.10, M S E=4.35$.

The critical test concerned the interaction between the identification variable and the linear trend contrast across the three parietal positions, indicating different left-right gradients for identified and unidentified words. This interaction effect was reliable, $F(1$, 29) $=83.85, M S E=0.84 .^{3}$ Decomposition showed that there was a linear trend for identified words, $F(1,29)=30.26, M S E=2.85$ (left $>$ center $>$ right). There was no such linear left-to-right trend for unidentified words. Indeed, there was a tendency toward a linear trend in the other direction (right $>$ left), $F(1,29)=3.42$, $M S E=1.91, p=.075$.

Although there was thus a lateralization difference for raw amplitudes, it remains to be determined whether the semantic priming effect changed its distribution. The three-way Category $\times$ Identification $\times$ Site interaction (linear trend) for the three parietal electrodes did not reach significance, $F(1,29)=3.04$, $M S E=0.18$.

As for Experiment 1, a more general test was also performed that involved all the recorded electrodes. Differences between in-category and out-of-category responses were computed, normalized, and entered into a 2 (identification) $\times 11$ (electrode sites) ANOVA. The Site $\times$ Identification interaction was not reliable, $F(10,290)=1.03, \epsilon=0.32, M S E=0.16$.

\section{Regression Test}

To test whether the $\mathrm{N} 400$ effect was more sensitive to category membership in unidentified words than was the binary-choice $d^{\prime}$, a regression analysis was performed. A derived variable was constructed to gauge the $\mathrm{N} 400$ effect, namely, the difference between in-category and out-of-category amplitudes for the $\mathrm{Cz}$ electrode. Figure 4 shows this variable regressed on the $d^{\prime}$ variable. Critically, the intercept (1.25) was reliably different from zero; $t(28)=2.50$. Similar results were obtained at the Pz site: intercept, $1.07, t(28)=2.25$. The slopes $(0.60$ and 1.35$)$ were not significantly different from zero $(t s<0.74)$.

\section{Discussion}

Experiment 2 replicated Experiment 1 in all important aspects. There was a semantic N400 effect among unidentified words. There were qualitative differences in the processing of unidentified compared with identified words, with respect to hemispheric asymmetries. The regression approach indicated an N400 effect even for the hypothetical case where behavioral categorization was precisely zero.

The signal-to-noise ratio in the electrophysiological recording was better in the second experiment than in the first, as a result of both the larger number of participants and of the more optimal balance of trials. The $\mathrm{N} 400$ effect was much larger among identified words in this experiment (about $4 \mu \mathrm{V}$ ) than in the first (about $1 \mu \mathrm{V}$ ), whereas there was no difference concerning the effect among unidentified words (both about $1 \mu \mathrm{V}$ ). The data pattern in Experiment 2, in which the effect for identified words was four times larger than that among unidentified words, was more in line with expectations. Possible reasons for the difference are discussed in a later section.

In contrast to the first experiment, sensitivity in behavioral categorization reliably exceeded zero, although the present average of 0.17 was very low. Still, the fact that it was above zero raises the question of whether the mental states driving this performance should be characterized as unconscious semantic processing or conscious processing of tacitly identified words. The first alternative requires that the end result of unconscious processing is made available to consciousness in shaping a deliberate response. The second alternative presupposes the withholding of oral responses, because of a high response criterion on the part of the participant, to words that have been consciously identified in their entirety. This second possibility is arguably strengthened by the finding that two-alternative, forced-choice (2-AFC) performance also exceeded chance, to an even higher degree than the semantic choice. The 2-AFC performance could, however, have a different explanation than full-word conscious identification. Recognition of single letters or parts of the word is sufficient to sustain performance in this task but not on the semantic-choice task. The need to address the issue of above-chance choice performance motivated a third experiment.

\section{Experiment 3}

In the two previous experiments, electrophysiological averaging was conditionalized on the oral identification of the words. Safeguarding against the possibility of participants' setting a high response criterion and tacitly performing unacknowledged reading has proceeded along several lines: encouragement to guess, the study of qualitative differences, and the use of binary-choice performance in the regression procedure. Still, it would be desirable to be able to base averaging on something other than a subjective verbal report. Neither of the two binary-choice measures in the previous experiment would fulfill the role, for both would result in many trials on which there was no conscious identification of the word being misclassified as being due to conscious identification because of the high prior probability of guessing on the correct alternative. Only a choice measure with a low prior probability would ensure that a reasonably small proportion of trials would be misclassified. Therefore, a multiplechoice task can be introduced in which the participant is given six alternatives among which to select the word that was displayed. In the absence of real perception, the participant has a one-in-six chance of selecting the right alternative, which inflates the number of identified trials somewhat but not seriously so. The effect on the ERP averages is to dilute the N400 effect among identified words by admitting a minor number of rogue trials into the fold of genuine ones. The critical comparison is, however, performed among unidentified words, where lucky guesses do not appear.

The six-alternative, forced choice (6-AFC) procedure goes a long way toward an exhaustive measure of conscious processing. Given that a conscious and veridical perception of a word had

\footnotetext{
${ }^{3}$ Rescaling, as recommended by McCarthy and Wood (1985), also resulted in a reliable effect, $F(1,29)=81.83, M S E=0.002$.
} 

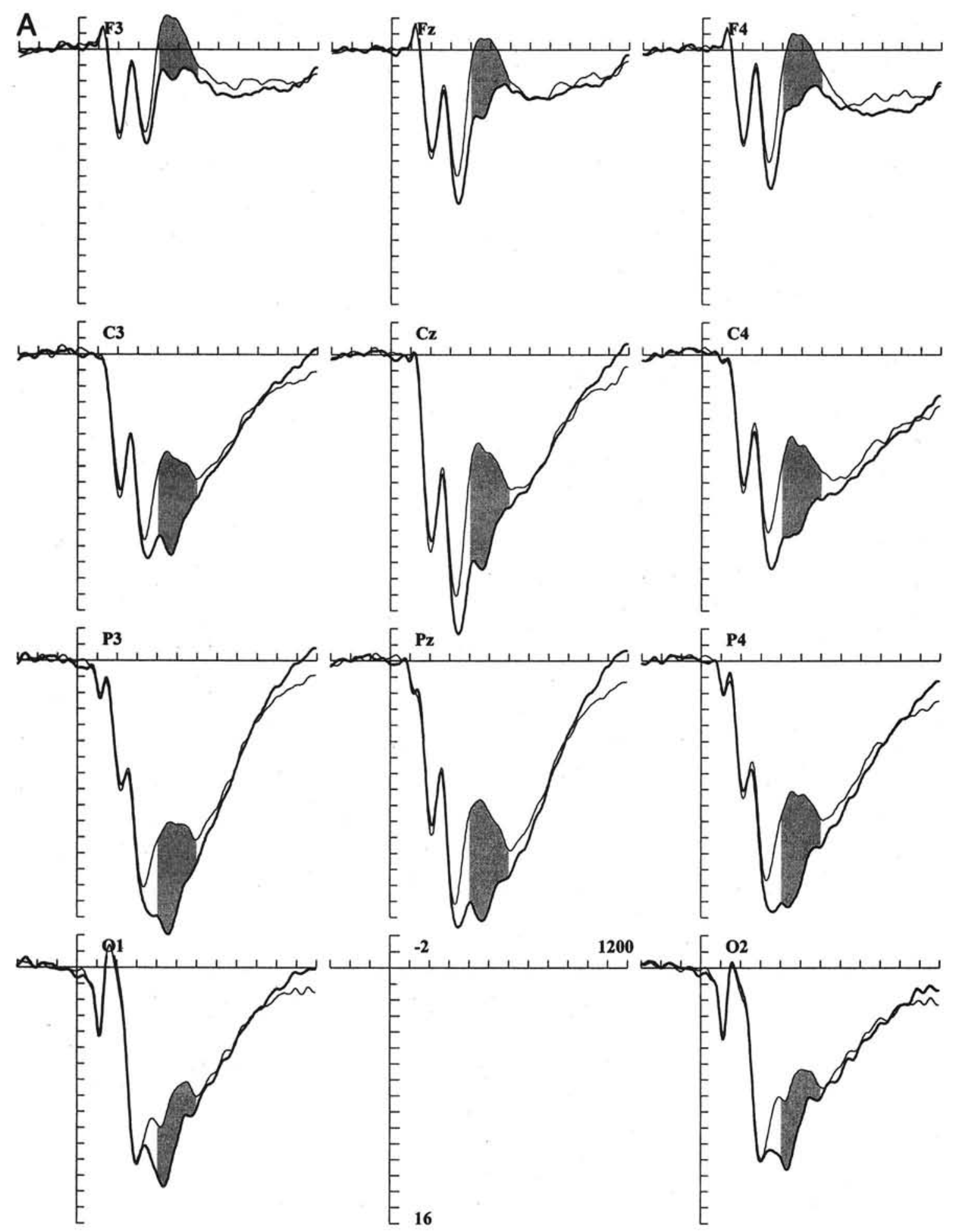

Figure 5A. Experiment 2: Event-related potential (ERP) wave forms for in-category (bold line) and out-ofcategory (thin line) words, plotted for identified words. The difference between in-category and out-of-category words is shaded in the critical time interval $(400-600 \mathrm{~ms})$. 

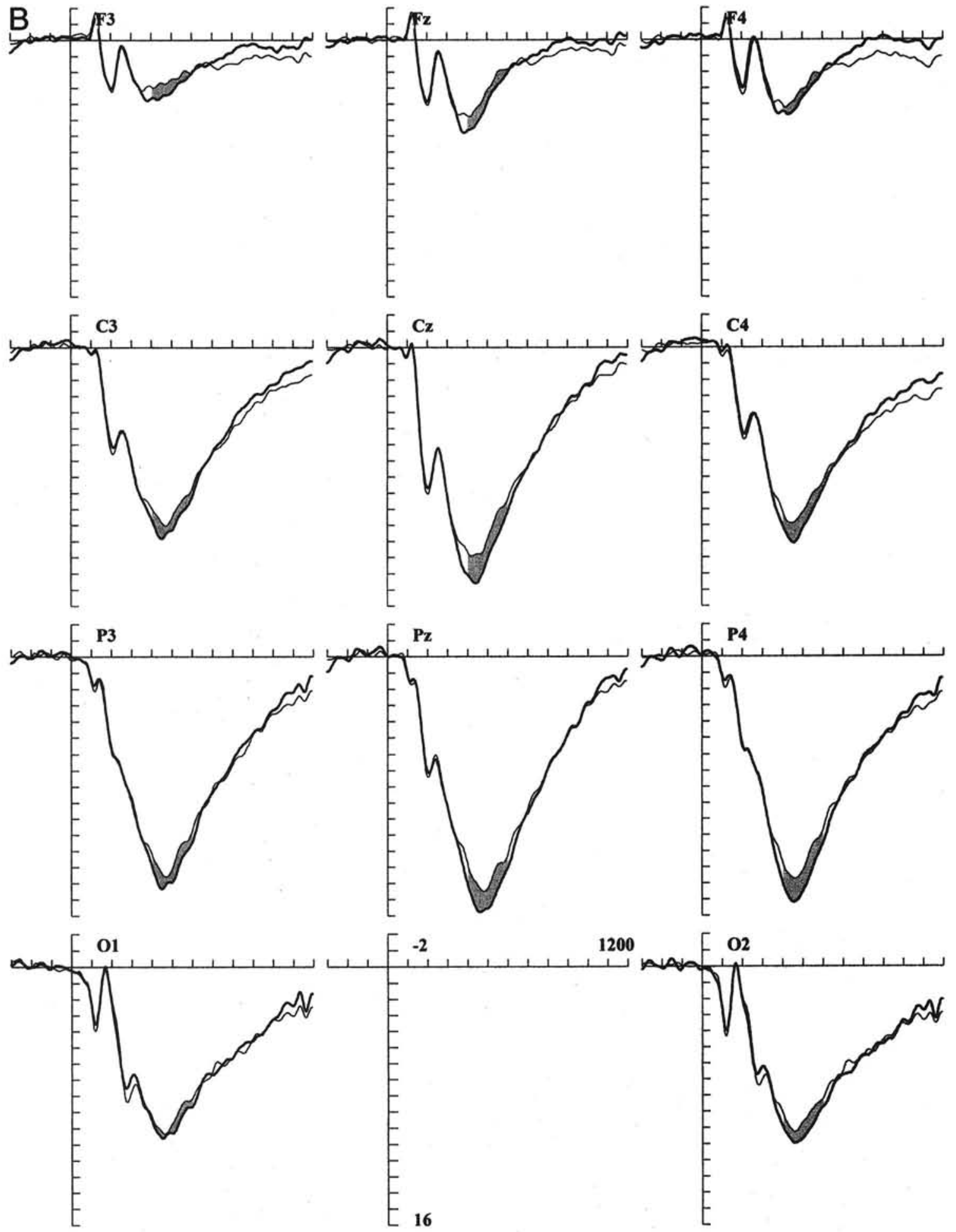

Figure SB. Experiment 2: Event-related potential (ERP) wave forms for in-category (bold line) and out-ofcategory (thin line) words, plotted for unidentified words. The difference between in-category and out-ofcategory words is shaded in the critical time interval $(400-600 \mathrm{~ms})$. 
arisen, it would be difficult to conceive of circumstances in which the correct alternative would be eschewed only a few seconds later. However, exhaustiveness was not our only concern. Hypothetically, unconscious processing may have led to an intuitive grasp of the word's meaning, and word alternatives presented immediately afterwards may have agreed to a greater or lesser extent with that memory trace, leading the participant to select among the alternatives accordingly. If we wish to make the task an exclusive test of conscious processing, selection on semantic grounds should not be possible, and only full awareness of the presented words should provide the key to selecting the correct alternative. Reducing the words to unique word fragments offered such a method. If, instead of the words orange, bicycle, carrot, knife, copper, and teacher, the fragments o_a_g_, b_c_c_e, c_r_o_, k_i_e, c_p_e_, and t_a_h_r are presented, participants would have little chance of making the choice on semantic grounds. Furthermore, the opportunity for basing the choice on single-letter or word-fragment recognition would be reduced when those letters or letter combinations were not presented in the test fragment.

\section{Method}

\section{Participants}

Thirty-five participants were tested and paid $200 \mathrm{kr}$ (\$24) each. One participant was excluded because of equipment malfunction, which resulted in lost data. Two participants were excluded because of abnormally low word identification rates (33\% and $35 \%$, both more than 3 SDs below the mean for the group). Of the remaining 32 participants, 15 were women. Mean age was 23 years (range $=19-35$ years). Two of the participants described themselves as left-handed.

\section{Recording}

Equipment, acquisition parameters, and postprocessing procedures were identical to those in Experiment 2, except for the addition of the $\mathrm{Oz}$ electrode to the set of recording sites (see Figure 1). Data from the $\mathrm{O} 2$ electrode were recorded, but because of a mistake in the amplifier settings, its filter differed from that of the other electrodes. Data from $\mathrm{O} 2$ were therefore not used.

\section{Stimulus Material}

A new set of 293 word triplets was constructed, each consisting of a category prime, a category member, and a category nonmember word. There were 79 categories in all (flowers, beverages, car parts, mammals, footwear, pets, spices, kitchen utensils, months, religions, languages, tools, etc.). The length of the stimulus words was uniformly in the range of 4-8 letters. They were all nouns, there were no proper names, and all words were shown in lowercase letters. In-category and out-of-category words were matched for length and size. (Listings and statistics are given on Web pages cited in Experiment 1.)

\section{Procedure}

Stimulus display, which was arranged as in Experiment 2, used three durations $(17,33$, and $50 \mathrm{~ms})$, the proportions of which were adjusted individually during the course of the experiment to approximate a $50 \%$ oral identification rate as closely as possible, using the same adaptive algorithm as before. Because the previous experiment had shown the intermediate level to be somewhat easier than intended, the contrast was lowered to $88 \%$. The other durations used full black-on-white $(97 \%)$ contrast.

Each trial proceeded as in Experiment 2, except that the display of two word alternatives was replaced by the display of six alternatives in a dialog window. In the six alternatives, every second letter was replaced by an asterisk (randomly starting with the first or the second letter). The distractor alternatives for each trial were constructed by random selection of five words from the remainder of the stimulus material, subject to the restriction that each of the six alternatives be unique after asterisk substitution.

\section{Results}

\section{Word Identification}

The adaptive testing procedure aimed at achieving an oral identification rate of approximately $50 \%$, with only minor variations among participants. The actual identification rate came very close to the target: $51 \%$, range $=46 \%-55 \%$. The procedure resulted in $32.4 \%$ of the words being shown at the shortest duration $(17 \mathrm{~ms}), 33.3 \%$ at the medium duration $(33 \mathrm{~ms})$, and $34.3 \%$ at the longest duration (50 ms).

As expected, duration affected identification rate very strongly, with $9 \%, 51 \%$ and $90 \%$ of the words identified at Levels 1,2 , and 3 . The probability of being correct after expressing confidence about word identification was .95 , whereas it was .55 after stating that the word was a guess. The abstention ("don't know") response was used in $34 \%$ of the trials and accounted for $69 \%$ of unsuccessful identification trials.

As before, a semantic priming effect arose from the category cue, such that the probability of identifying a word that belonged to the cued category was .57 versus .45 for a miscued word. This overall priming effect $(0.12)$ was greatest at the intermediate level $(0.20)$ and smaller at the difficult $(0.12)$ and the easy levels $(0.05)$.

\section{6-AFC Performance}

Overall, the proportion of trials on which the correct word fragment was chosen was .656. Among orally unidentified words, the probability of correct fragment choice was .307 (range $=.160-.419$ ). Tested against the expected guessing level of $1 / 6$, performance clearly exceeded chance, $t(31)=13.60$. Correct fragment choice for orally unidentified words rose as a function of exposure duration: $0.23,0.38$, and 0.63 for Levels $1-3$, respectively.

\section{Binary Categorization Performance}

Signal-detection sensitivity was computed for the binary choice of whether the word belonged to the cued category or not. This computation was, as before, performed for unidentified words only. Conditionalizing this on either oral identification or fragment-choice identification gave different results.

With an oral criterion (subjective threshold), average $d^{\prime}$ was 0.228 , with a $95 \%$ confidence interval from 0.114 to 0.341 . The mean was significantly different from zero, $t(31)=4.09$.

With a fragment-choice criterion (objective threshold), average $d^{\prime}$ was 0.040 , with a $95 \%$ confidence interval extending from -0.079 to 0.159 . The mean was not different from zero, $t(31)=0.69$.

\section{$E R P_{S}$}

The four-cell averaging was performed in two ways: based on verbal report identification (as before) and based on 6-AFC identification. In the following, both sets of results are presented. (In 
verbal report averaging, the number of trials in the cells [after artifact rejection] were as follows [ $M \pm S D$ ]: Identified/InCategory [IdIn], $66 \pm 5$; IdOut, $52 \pm 7$; UnidIn, $51 \pm 4$; and UnidOut, $65 \pm 6$. The corresponding numbers in 6-AFC-based averaging were IdIn, $81 \pm 7$; IdOut, $71 \pm 6$; UnidIn, $35 \pm 6$; and UnidOut, $45 \pm 5$. The minimum number of trials in any cell, for any participant, was 24.) Waveforms for the midline electrodes are plotted in Figure 6A and Figure 6B (verbal) and in Figure 7A and Figure 7B (6-AFC).

Verbal report identification. As before, mean amplitudes in the 400-600-ms interval for three midline electrodes were analyzed in a three-way ANOVA. The variables were word identification, category membership, and electrode site.

The category main effect was reliable, $F(1,31)=81.69$, $M S E=9.18$, as was the Category $\times$ Identification interaction, $F(1,31)=30.31, M S E=7.45$. Simple main effects showed a large category effect for identified words, $F(1,31)=86.16$, $M S E=10.44, \eta^{2}=0.74$. Deviations from the expected category reduced mean amplitude substantially, from 10.4 to $6.1 \mu \mathrm{V}$.

Crucially, the same effect emerged for unidentified words. The category effect was significant, $F(1,31)=12.34, M S E=6.19$, $\eta^{2}=0.29$. The amplitude reduction was smaller but reliable, from 10.5 to $9.2 \mu \mathrm{V}$.

6-AFC identification. A similar three-way analysis showed the category main effect to be reliable, $F(1,31)=67.96$, $M S E=8.29$. There was a Category $\times$ Identification interaction, $F(1,31)=25.63, M S E=6.97$. The simple main effect of category on identified words was reliable, $F(1,31)=85.47$, $M S E=8.05, \eta^{2}=0.73$. The critical category effect among unidentified words was also reliable, $F(1,31)=7.45$, $M S E=7.21, \eta^{2}=0.19$. The $1.3-\mu \mathrm{V}$ effect in the orally defined data was marginally diminished to a $1.1-\mu \mathrm{V}$ effect in the 6-AFC data, from 11.0 to 9.9 .

\section{Qualitative Differences}

Verbal report identification. Data from the parietal electrodes were tested in a three-way ANOVA (Identification $\times$ Category $\times$ Lateral Site). As expected, there were effects of category, $F(1$, 31) $=104.78, M S E=7.94$, and a Category $\times$ Identification interaction, $F(1,31)=43.24, M S E=6.96$, and there were simple main effects of category among identified words, $F(1,31)=$ $121.28, M S E=8.80$, as well as among unidentified words, $F(1$, 31) $=10.82, M S E=6.11$.

Most important, the interaction between the identification variable and the linear trend across the parietal sites was reliable, $F(1$, $31)=49.06, M S E=1.14 .^{4}$ There was a linear trend from left to right for identified words, $F(1,31)=19.04, M S E=4.68$, but there was none for unidentified words, $F(1,31)=0.42, M S E=3.10$ (see Figure 8).

Testing instead the distribution of the semantic priming effect, the three-way Category $\times$ Identification $\times$ Site interaction (linear trend) for the three parietal electrodes did not reach significance, $F(1,31)=2.92, M S E=0.16$. Similarly, taking all 11 electrodes into account, rescaled differences between in-category and out-ofcategory responses did not give a reliable Site $\times$ Identification interaction, $F(10,310)=1.86, \epsilon=0.35, M S E=0.13$.

6-AFC identification. In a similar analysis, using 6-AFC as the identification criterion, there were effects of category, $F(1$, 31) $=88.85, M S E=7.15$, and a Category $\times$ Identification interaction, $F(1,31)=34.14, M S E=6.91$, and there were simple main effects of category among identified words, $F(1,31)=$ $113.80, M S E=7.23$, as well as among unidentified words, $F(1$, 31) $=7.11, M S E=6.83$.

The interaction between identification and the linear trend was reliable, $F(1,31)=40.45, M S E=1.14$. $^{5}$ There was a linear trend from left to right for identified words, $F(1,31)=15.53$, $M S E=3.86$, but not for unidentified words, $F(1,31)=1.02$, $M S E=3.42$.

The semantic priming effect, on the other hand, did not change its scalp distribution, as reflected in a nonsignificant Category $\times$ Identification $\times$ Site (linear trend) interaction, $F(1,31)=0.65$, $M S E=0.24$. Likewise, the priming-related differences over all 11 electrodes gave no reliable Site $\times$ Identification interaction, $F(10$, 310) $=1.25, \epsilon=0.44, M S E=0.16$.

\section{Regression Test}

The $\mathrm{N} 400$ effect (i.e., the difference due to category membership in the 400-600-ms interval) was regressed on behavioral categorization sensitivity. Using the oral identification criterion, the intercept was found to be 0.94 , not quite reliably different from zero, $t(30)=1.81, p=.08$. A similar intercept $(0.92)$ was found for the $\mathrm{Pz}$ site, $t(30)=1.81, p=.08$. Slopes 1.84 and 1.42 were not significant (both $t \mathrm{~s}<1.36$ ).

Using the 6-AFC criterion, intercepts were found, however, to be reliably different from zero. At $\mathrm{Cz}$, the intercept was 1.07 . $t(30)=2.32$, and at $\mathrm{Pz}$ it was $1.04, t(30)=2.50$. Slopes 1.44 and 1.24 were not significant (both $t \mathrm{~s}<1.03$ ). (See Figure 9.)

\section{Discussion}

The main objective of Experiment 3 was to separate the definition of identified-unidentified trials from its reliance on a subjective criterion (i.e., the one involved in selecting whether to respond with an oral identification). The six-alternative, forced-choice procedure introduced a bias-free response method, and its results could be compared with those of the verbal response.

Thirty percent of words unidentified by verbal report were correctly identified in the forced-choice task. Several sources may have contributed to this figure, and lucky guesses is one of them. If we estimate the chance of guessing correctly as $1 / 6$, then $14 \%$ $(30.7 \%-16.7 \%)$ remained to be explained by other sources. Tacit reading (i.e., conscious identification suppressed by a strict oral response criterion) is the source of greatest theoretical interest for present purposes, but it is not the only one. Perceptually based identification is also possible, in the absence of full-word reading. The participant may have noticed that the word started with the letter $t$, and this clue may have been sufficient to identify the correct word fragment in the 6-AFC task. In the present experiment, it was not possible to estimate how great the contribution of perceptual, presemantic identification was, although in principle, it could have been determined by varying the proportion of intact letters in the word fragments.

The extent of tacit reading can be approached from a different vantage point. Among orally unidentified words, the binary cate-

\footnotetext{
${ }^{4}$ With McCarthy-Wood rescaling, $F(1,31)=50.09 ; M S E=0.002$.

${ }^{5}$ With McCarthy-Wood rescaling, $F(1,31)=42.50 ; M S E=0.002$.
} 

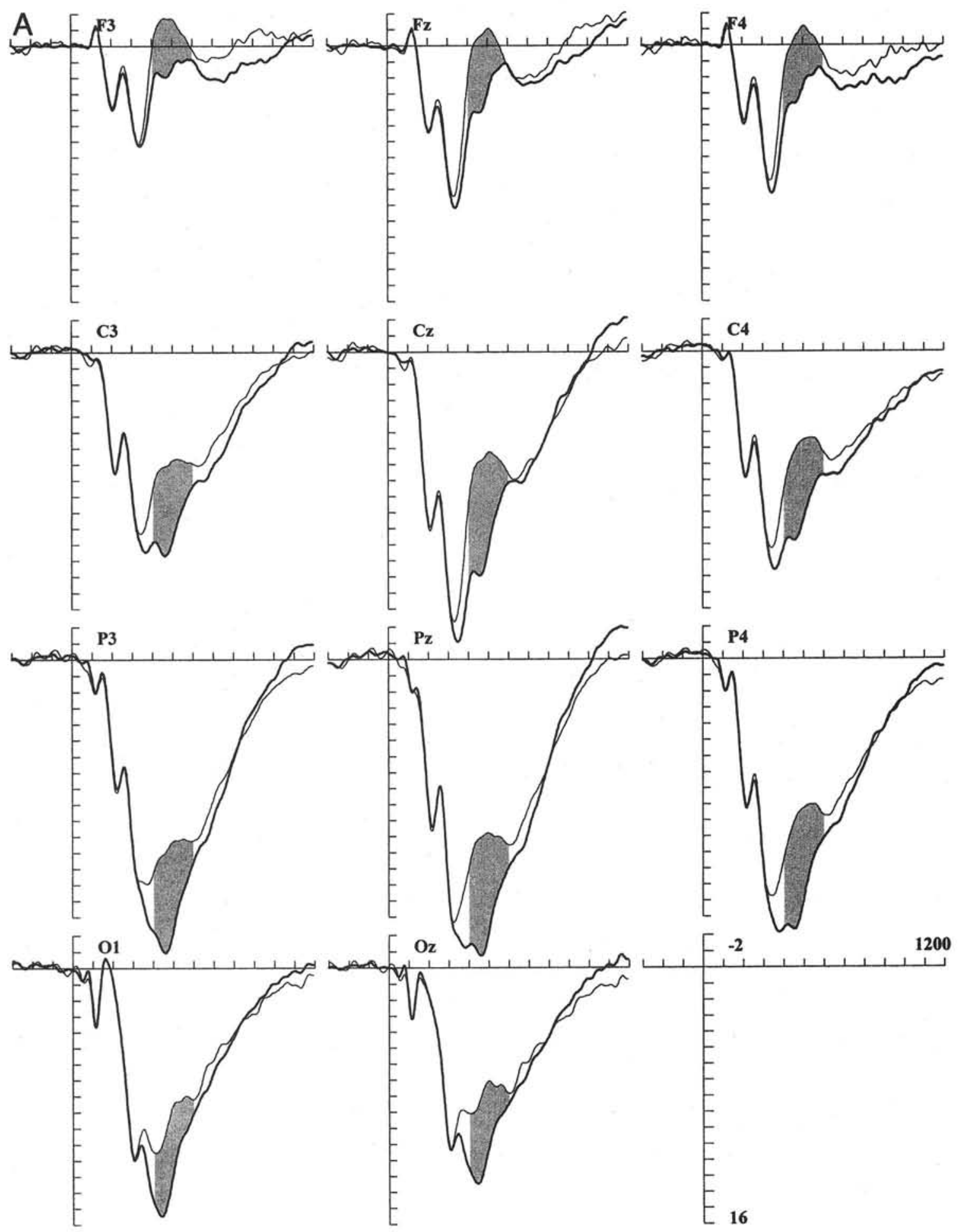

Figure 6A. Experiment 3, verbal report: Event-related potential (ERP) wave forms for in-category (bold line) and out-of-category (thin line) words, plotted for identified words. The difference between in-category and out-of-category words is shaded in the critical time interval $(400-600 \mathrm{~ms})$. 

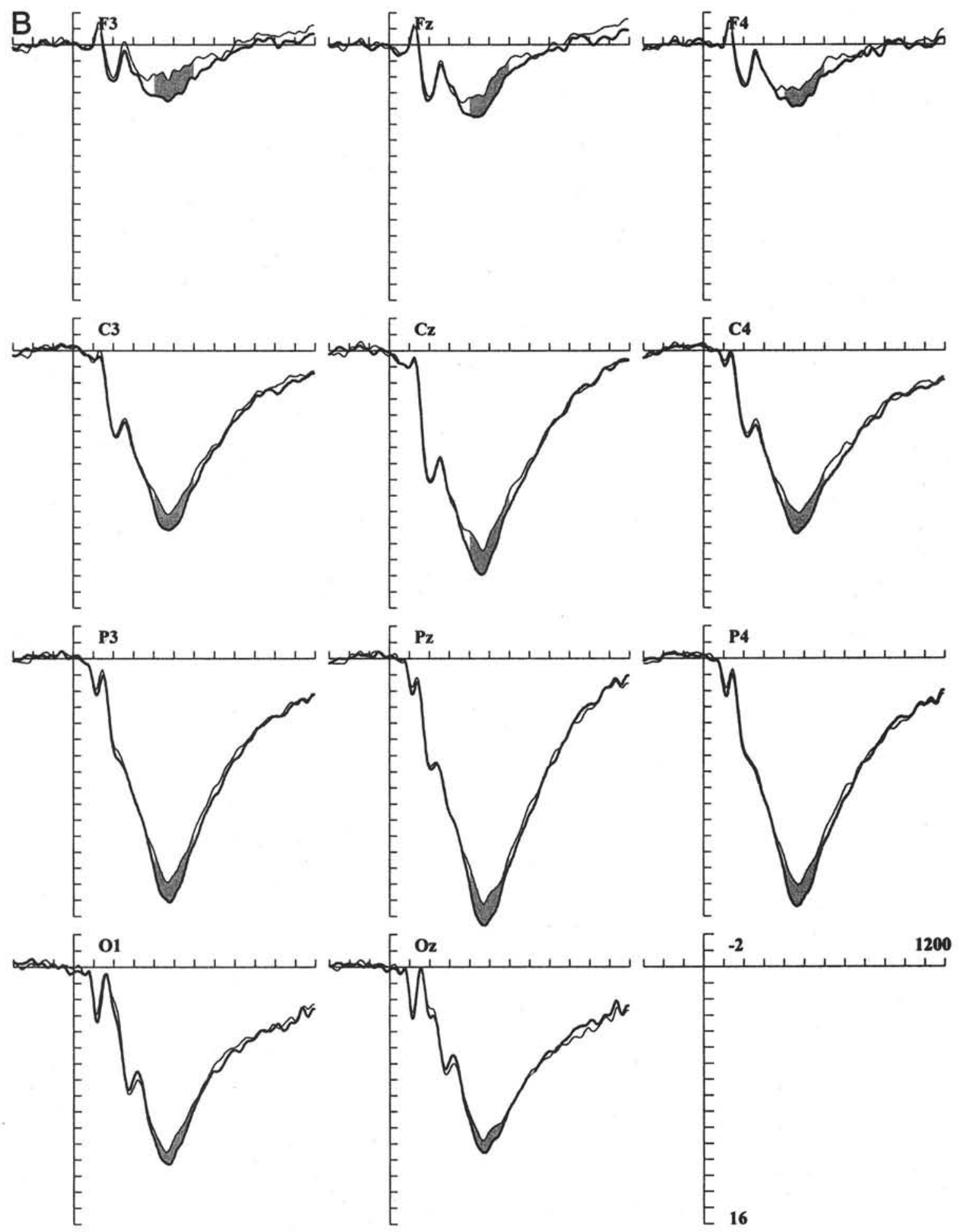

Figure 6B. Experiment 3, verbal report: Event-related potential (ERP) wave forms for in-category (bold line) and out-of-category (thin line) words, plotted for unidentified words. The difference between in-category and out-of-category words is shaded in the critical time interval $(400-600 \mathrm{~ms})$. 

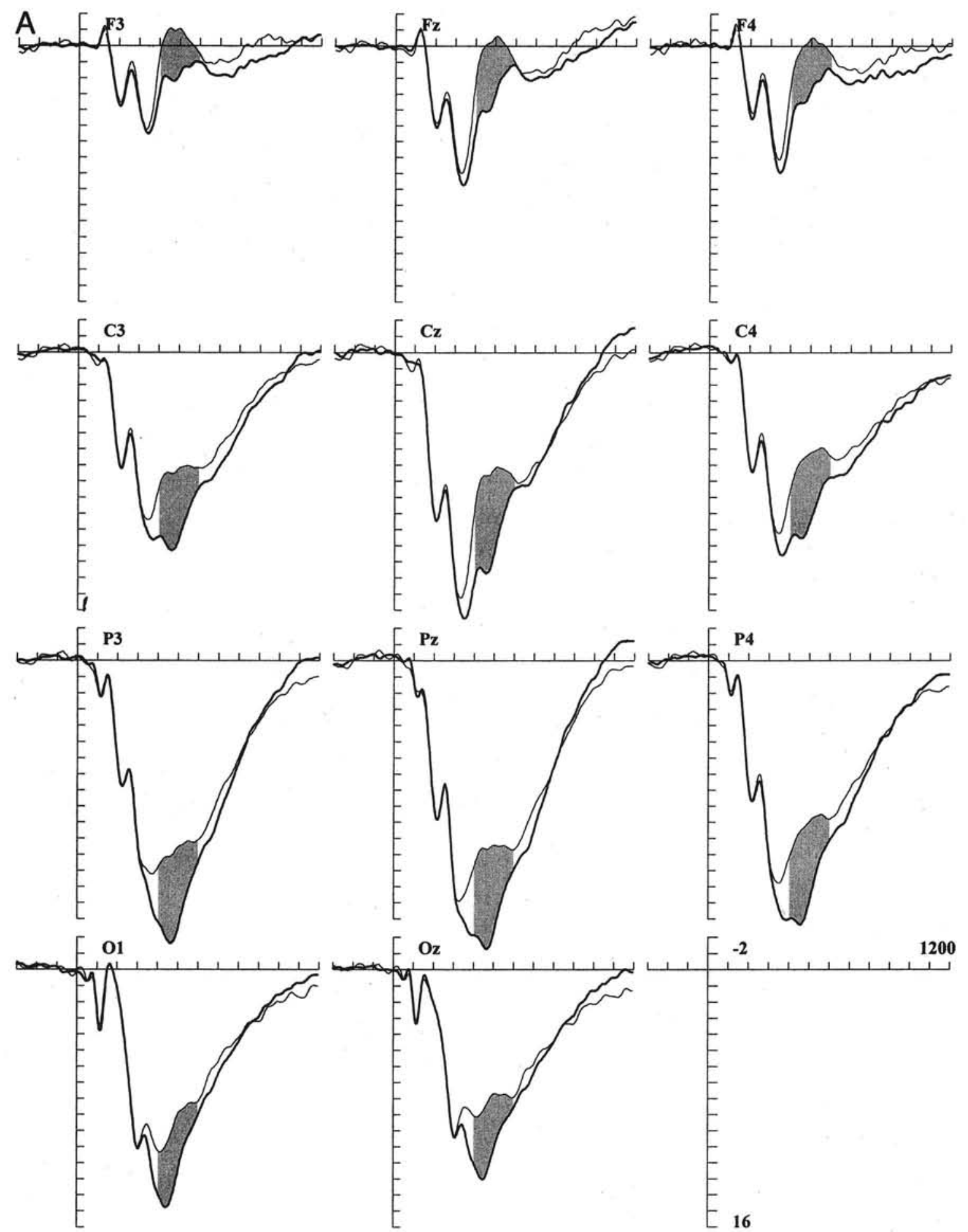

Figure 7A. Experiment 3, 6-AFC definition: Event-related potential (ERP) wave forms for in-category (bold line) and out-of-category (thin line) words, plotted for identified words. The difference between in-category and out-ofcategory words is shaded in the critical time interval $(400-600 \mathrm{~ms}) .6-\mathrm{AFC}=$ six-alternative forced choice. 


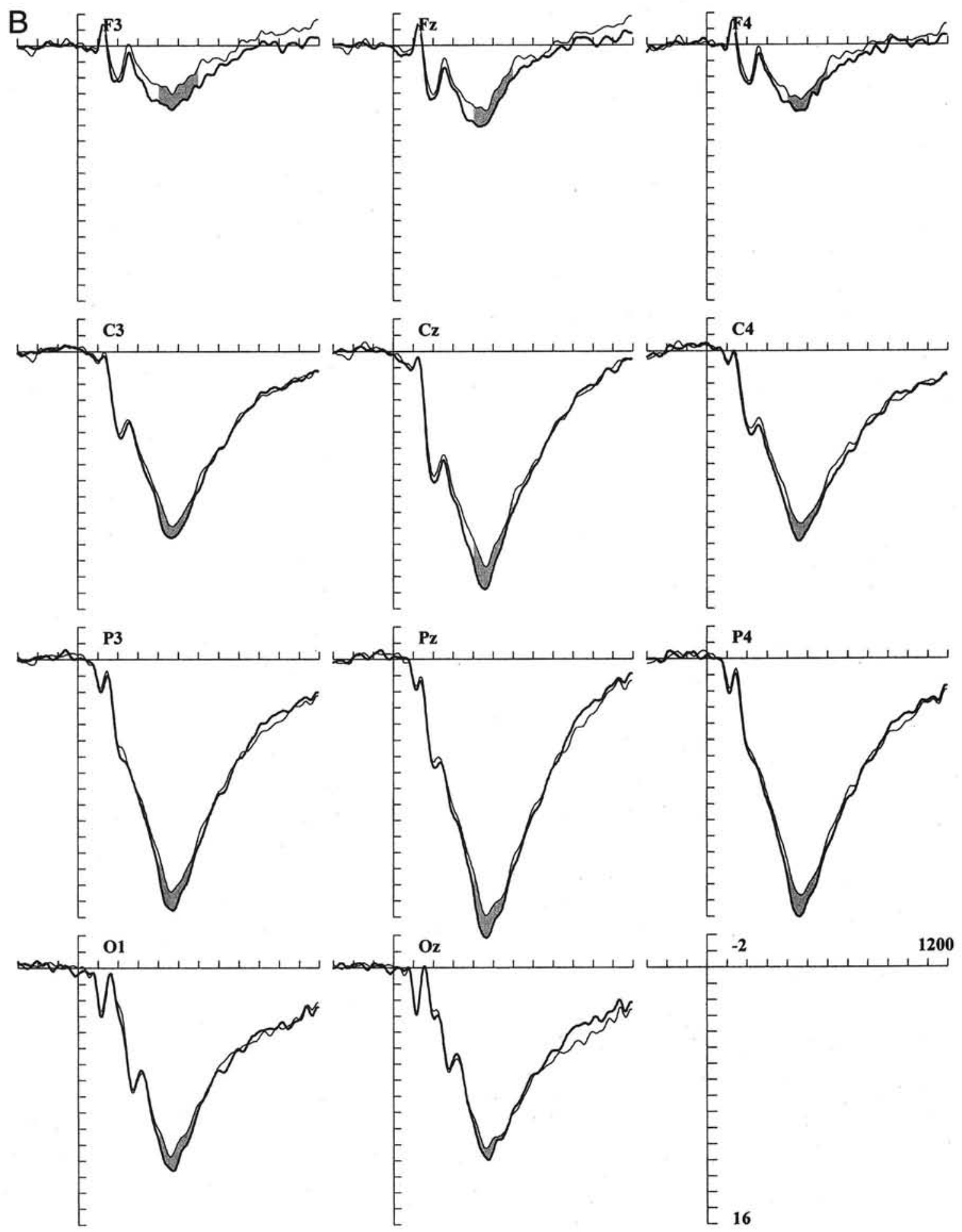

Figure 7B. Experiment 3, 6-AFC definition: Event-related potential (ERP) wave forms for in-category (bold line) and out-of-category (thin line) words, plotted for unidentified words. The difference between in-category and out-of-category words is shaded in the critical time interval $(400-600 \mathrm{~ms}) .6-\mathrm{AFC}=$ six-alternative forced choice. 

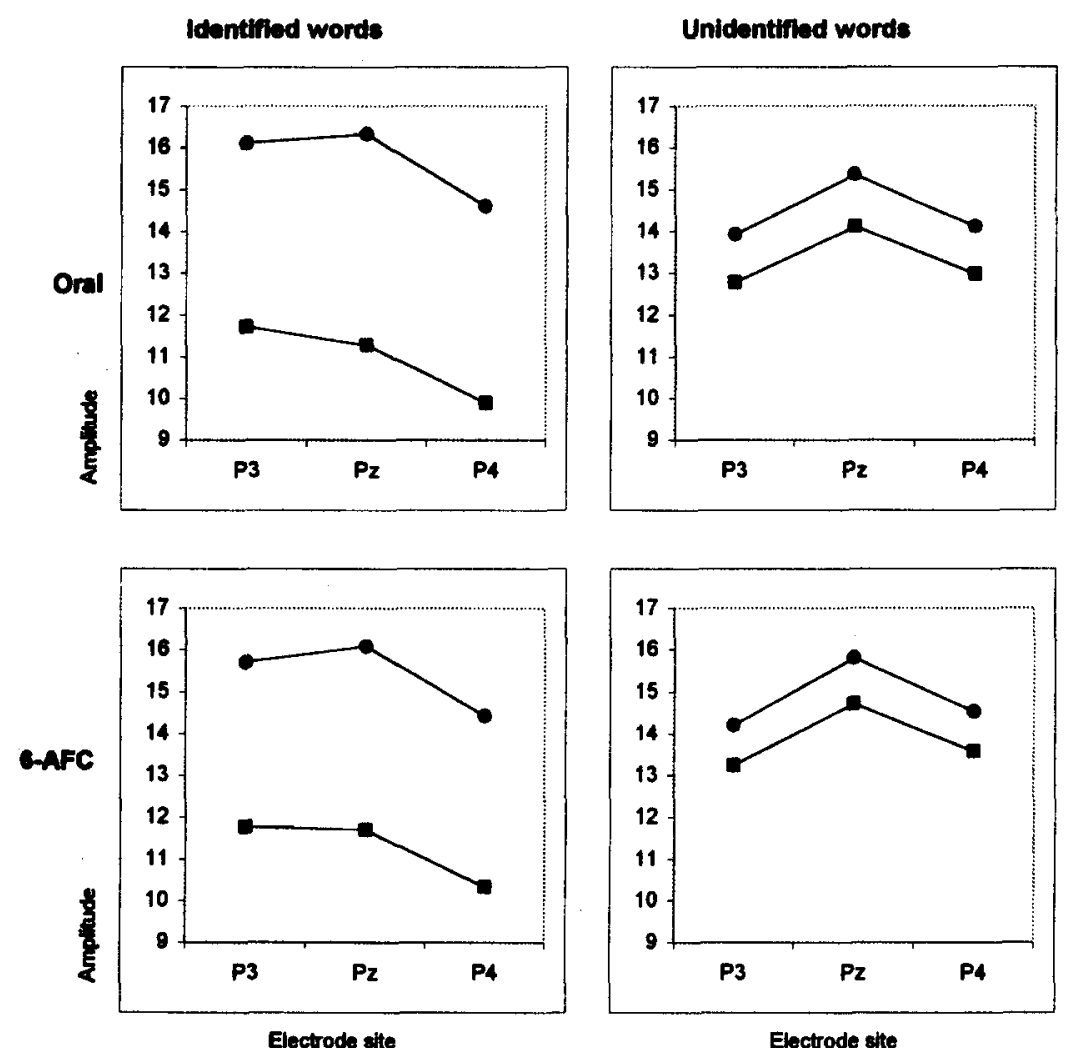

Figure 8. Experiment 3: Mean amplitudes in the 400-600-ms band at three parietal sites. Round markers show in-category words, and square markers show out-of-category ones. The upper row shows identification defined by oral report, and the lower row shows six-alternative forced choice (6-AFC).

gorization task was performed with above-zero accuracy. The $d^{\prime}$ value was 0.23 , corresponding to 55\% correct. Partitioning the set of orally unidentified words into those for which 6-AFC was correct and those for which it was incorrect made a great difference for binary categorization performance. In the subset where both types of identification were unsuccessful, binary categorization performance was at chance (51\% correct). In the subset where 6-AFC was successful, categorization performance was better than chance ( $64 \%$ correct). Thus, there seem to be grounds for believing that some tacit reading occurred. The categorization performance could not be supported by presemantic fragmentary perception alone. Perhaps the proportion of tacit reading could go as high as $13 \%-14 \%$ of orally unidentified words.

Still, for all the serious consideration that the problem of tacit reading has to be given, the data are clear in exonerating it of causing the semantic ERP effects in unidentified words. After elimination of all trials where participants chose the right 6-AFC alternative, there were still different ERPs to category and noncategory words. The effect was almost as large in the 6-AFC as in the verbal report data $(1.1 \mu \mathrm{V}$ compared with $1.3 \mu \mathrm{V})$. Other aspects of our earlier findings also withstood the test of a stricter objective selection criterion. A different, laterally symmetric pattern of ERP amplitudes characterized the processing of unidentified, as opposed to identified, words, and this held true below the objective threshold as well as below the subjective threshold. The intercept for the function relating the ERP effect to binary categorization performance was reliably above zero for objectively unidentified words.

Our concerns that the semantic ERP effect might depend on tacit, unacknowledged reading proved untrue. Semantic content shaped the brain responses to unconsciously perceived words, and this seemed to hold true regardless of whether consciousness was defined in relation to an objective or a subjective threshold.

\section{General Discussion}

This study has shown evidence of high-level word processing in the absence of conscious identification. Masked words, about which participants professed ignorance, evoked brain responses that were diagnostic of the words' semantic qualities. Words belonging to a designated category elicited brain potentials different from those of words outside of the category, and we attribute the difference to semantic processing without awareness.

\section{The Psychophysiological Indicator}

The variable that we found to differentiate between words according to their semantic qualities was mean amplitude in the 400-600-ms range. There seem to be good prima facie reasons for identifying this with the $\mathrm{N400}$, which was originally discovered in a sentence-processing task (Kutas \& Hillyard, 1980). It has been well established that the $\mathrm{N} 400$ can be produced in semantic prim- 


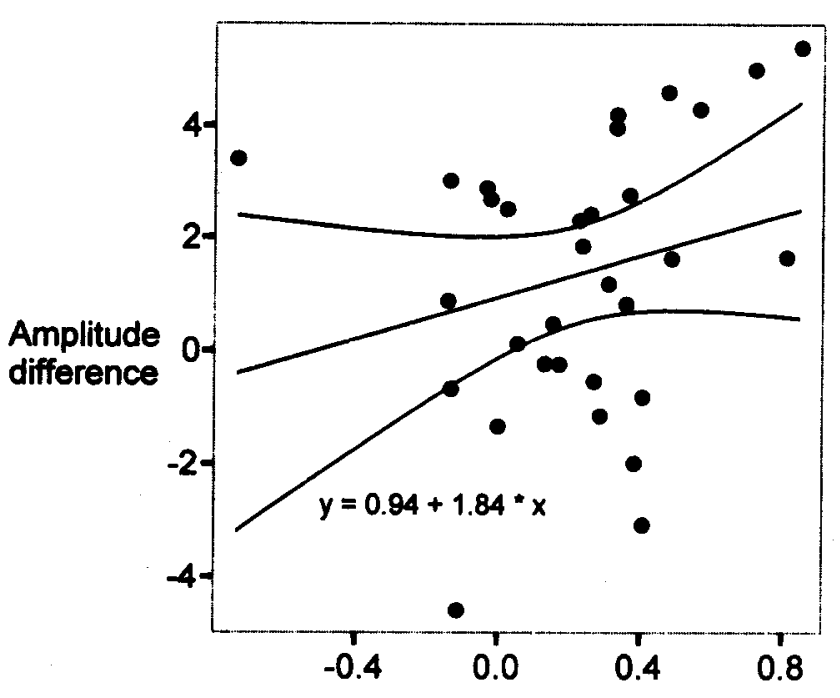

$d^{\prime}$

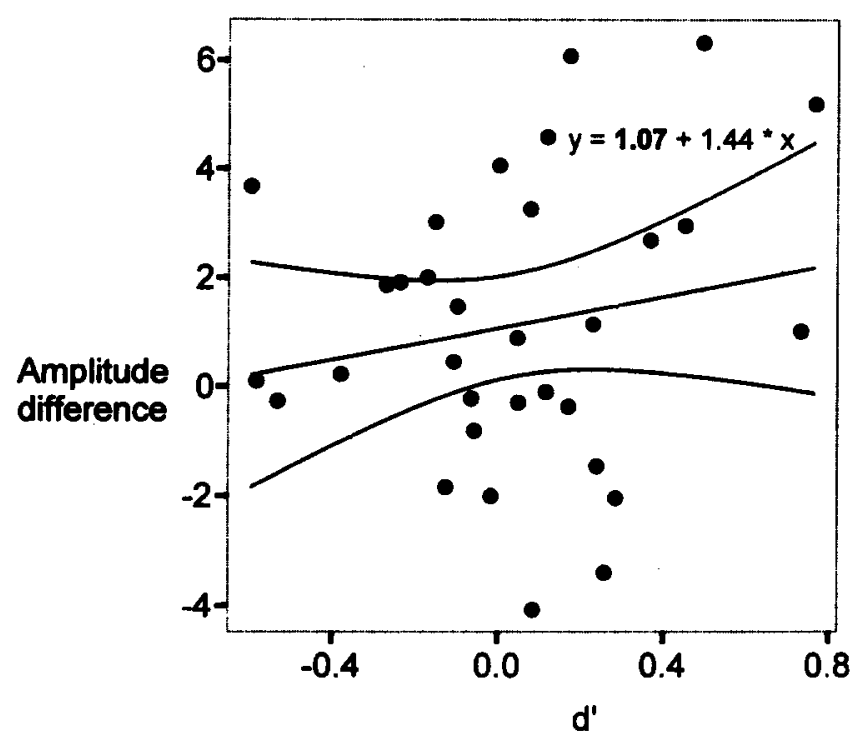

Figure 9. Experiment 3: Scatterplots with regression lines and $95 \%$ confidence intervals (curved lines). In both panels, the ordinate shows the amplitude difference between in-category and out-of-category words at the $\mathrm{Cz}$ site for unidentified trials, and the abscissa shows $d^{\prime}$ in semantic binary choice for unidentified words. Top: identification defined by verbal report. Intercept shows marginal tendency to differ from zero $(p=.08)$. Bottom: identification defined by six-alternative forced choice (6-AFC), intercept reliably different from zero. Boldface type is used in regression equations for intercepts or slopes that are significantly different from zero.

ing tasks using isolated words, not sentences, as stimuli (e.g., Bentin et al., 1985). The topography of the semantic effect agrees well with what is known about the N400, with a centro-parietal maximum for the difference between in-category and out-ofcategory words. However, the typical wave shape of the $\mathrm{N} 400$, with a well-defined peak, was found only in the above-threshold ERPs. The subthreshold ERPs showed a smaller effect with a less characteristic wave shape. The weaker effect for unidentified words, which was more probabilistic in its occurrence and latency, was probably responsible for the atypical wave form. Comparisons of the difference waves (Figure 10) show the effects for identified and unidentified words to be located in the same time interval. As to localization, tests of the scalp distribution of the semantic priming effect revealed no influence of word identification.

Although an argument built on the absence of significant differences cannot be completely compelling, the conclusion to be tentatively drawn from our findings of similar distributions in time and space is that we are dealing with the same phenomenon, below threshold as well as above it. If, on the other hand, we were to be moved by the uncharacteristic wave shape to withhold the name of N400 and refer to it as a hitherto unknown component, very little would be changed. Our purpose was to examine ERP differences between words on the basis of their semantic characteristics. Any such difference found among unidentified words is relevant for the question of semantic processing without awareness. Connecting the effect with the $\mathrm{N} 400$, as known from the literature, enhances our understanding of it, but without that support, it would still be an empirical finding with direct bearing on our research question.

The validity of the argument rests on there being no other differences, besides semantic ones, between in-category and out-ofcategory words that could explain the differences in psychophysiological response. It is a weakness of the present study that we used different sets of words for the in-category and out-of-category stimuli. To rule out potential item effects, the stimulus words need to be examined. It is a large pool of words-1,542 Swedish words with their category primes. Materials for the three experiments were primarily constructed independently of each other, and the overlap was relatively small—in all, 1,152 different words were used. They can be viewed, along with their English translations and other data (physical measures, frequency, part of speech, category, etc.), on the Internet at http://www.psychology.lu.se/Personal/Stenberg/SemanticERPs/.

First and foremost, it is important to determine whether there were any differences in physical appearance between category members and nonmembers, because if there were, the two types of word could be distinguished without recourse to meaning, and ERP differences between them might be explained by simple sensory processing. The physical characteristics of the words were compared in several respects: word length (number of letters), on-screen word width (in pixels), and total on-screen word size (number of pixels occupied by the word). All differences between category members and nonmembers were small and nonsignificant. (The figures are given on the Web pages.)

Word frequency is a characteristic of a different sort. Although it is not as immediately obvious that it can play a part in early processing, some models of word recognition do assume that a frequency-ordered search precedes lexical access. Therefore, it is at least possible that frequency could affect the ERPs even if no conscious identification has taken place. Word frequencies in the stimulus material were assessed with the help of a large, Internetaccessible database of printed Swedish (http://spraakdata.gu.se/lb/ konk/). Statistics for our stimulus words are given on our Web pages. Tests were computed using the $\log$ transform of the 
$\operatorname{Exp} 1$

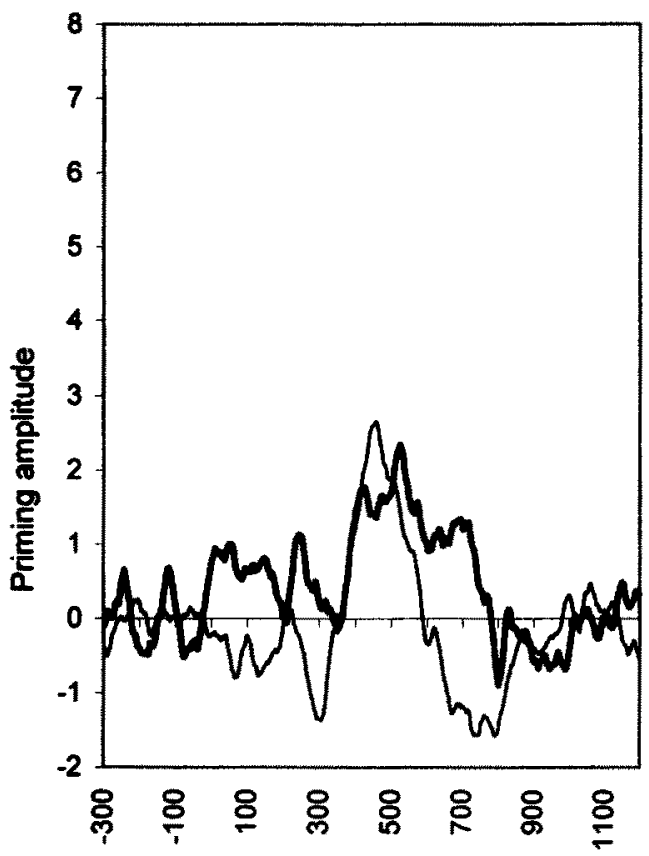

Exp 3 - verbal rep

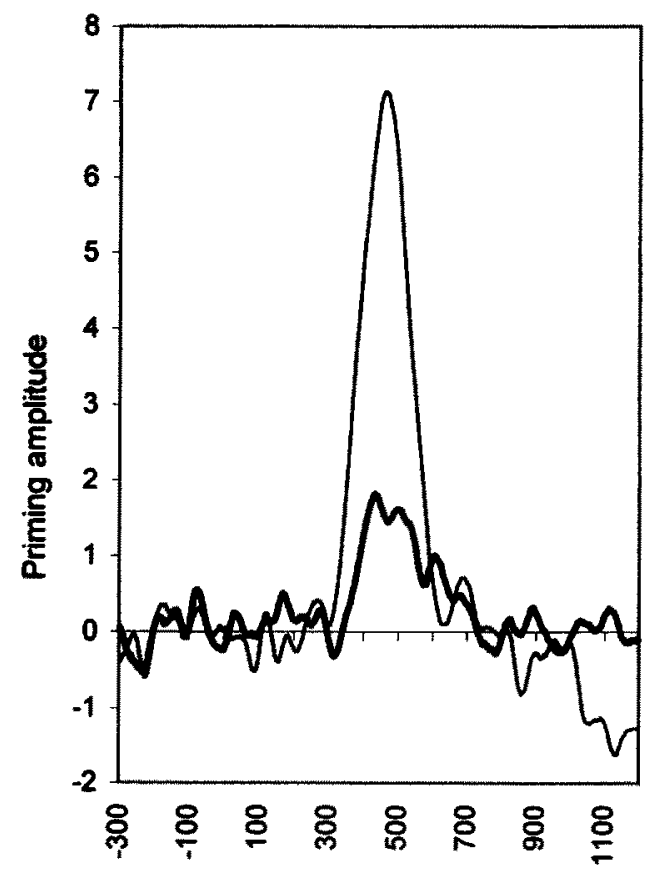

Exp 2

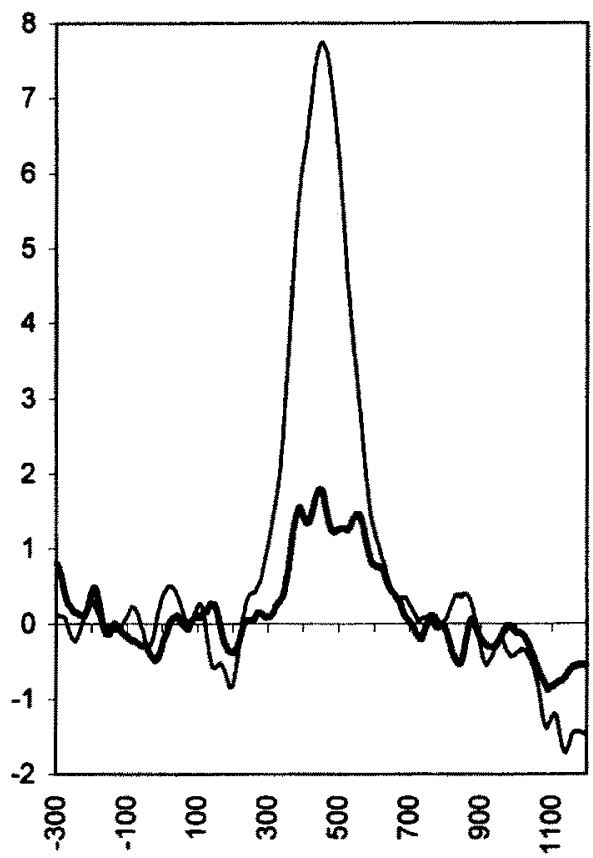

Exp 3 - 6AFC

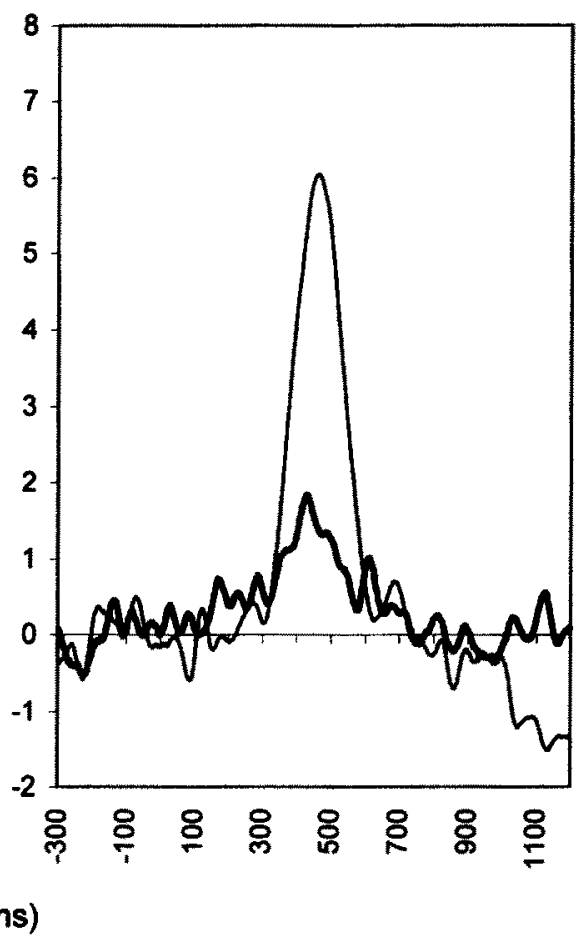

Figure 10. Difference waves, formed by subtraction of out-of-category from in-category words. Thin lines refer to identified words and bold lines to unidentified ones. The figure shows data from all three experiments. Exp $=$ experiment; verbal rep $=$ verbal report; $6 \mathrm{AFC}=$ six-alternative forced choice. 
(skewed) frequency variable, and the means given in the following paragraph have been inverse transformed back to the original metric.

There were significant differences between in-category and outof-category words in all three experiments, although in different directions. Category members were less frequent than nonmembers in Experiment 1 (5.69 vs. 57.06 occurrences per million [OPM]), more frequent in Experiment 2 (10.57 vs. 7.68 OPM), and again less frequent in Experiment 3 (6.66 vs. 12.38 OPM). Consider, for example, Experiments 2 and 3 . The results in these were very similar, as were the ERP wave shapes; but the frequency differences went in opposite directions. It is difficult to imagine that frequency could have caused the ERP differences between in-category and out-of-category words when they were thus dissociated.

In the literature, the impact of frequency on the $\mathrm{N} 400$ is that low-frequency words give larger N400s (Van Petten \& Kutas, 1990; Young \& Rugg, 1992). In our experiments, that influence runs counter to the results of Experiments 1 and 3, in which the higher frequency out-of-category words showed larger N400s. Therefore, it seems very unlikely that a frequency confound could have caused the category membership effects on the ERPs.

In other grammatical and semantic respects, our stimulus material was mostly homogeneous. A large majority of the words $(97 \%)$ were nouns. It was more difficult to produce statistics for semantic variables, such as concreteness, because we had no rating norms for Swedish words at our disposal. As to the concreteness dimension, informal inspection suggests that almost all the words are concrete, with the exception of a certain number of the outof-category words in Experiment 1. Other semantic variables, such as number of associations and number of meanings, are more difficult to assess informally.

It is a different question as to what impact these variables may have had on our main hypothesis. Our main claim is that we have evidence for semantic processing of words that are not consciously identified. Consider a hypothetical case in which there would be systematic semantic differences between our two classes of words, apart from the intended category membership distinction. It could then be claimed that participants unconsciously processed, not category membership, but, say, concreteness of the words. The data would still show semantic processing without awareness.

Of possible semantic variables, concreteness is probably the best known from the electrophysiological literature. Kounios and Holcomb (1994) have shown larger N400s for concrete than for abstract words. Again, that would have worked against us in obtaining our results, assuming that the concrete-abstract imbalance in Experiment 1 makes a difference. Because some of the out-of-category words in that experiment were more abstract, the concreteness dimension should have counteracted the effect we found (i.e., that out-of-category words had larger $\mathrm{N} 400 \mathrm{~s}$ ). In any case, an influence from semantic item characteristics could only act through semantic processing of unidentified words, and therefore it would not contradict the main conclusion of this study.

\section{Other N400 Findings}

In a general sense, support can be found for the present findings in the $\mathrm{N} 400$ literature, particularly in those studies indicating automatic processing as the generating force behind the component. To these belong studies showing priming at short SOAs (Anderson \& Holcomb, 1995) as well as a degree of independence of task (Besson et al., 1992; Kutas \& Hillyard, 1989) and attention (Brualla et al., 1998). On the other hand, a number of other studies indicate the importance of attention (Holcomb, 1988; McCarthy \& Nobre, 1993) and of having a semantically oriented task (Chwilla, Brown, \& Hagoort, 1995). The literature suggests that controlled processing, if not necessary, is at least influential in producing the $\mathrm{N} 400$ and that "the N400 component reflects both automatic and controlled aspects of priming" (Anderson \& Holcomb, 1995, p. 189).

In the special case of studies investigating the $\mathrm{N} 400$ as a reflection of priming with brief, pattern-masked stimuli, the findings have been negative (Brown \& Hagoort, 1993; Münte \& Heinze, 1991), in contrast to the present results. The contradiction between our results and those of the earlier studies, however, may be more apparent than real. The important aspect in which the present study differs from those of Brown and Hagoort (1993) and Münte and Heinze (1991) is that we used a suprathreshold prime and a marginally perceptible target, which is the reverse of the standard procedure. In priming studies, the semantic effect, of which the $\mathrm{N} 400$ is an indicator, results from the combined action of prime and target. The prime imparts activation to the word node belonging to the target. This activation can be conceived as an elevation of the resting activation level (Collins \& Loftus, 1975), or equivalently as a lowering of the criterion threshold that needs to be surmounted for the node to fire (Morton, 1969). When the target is displayed, activation derived from perceptual processing flows to the target node, where it is added to the resting activation level. Presumably, the relation between incoming activation and the resulting activity level of the node is a nonlinear, sigmoid one, as shown in Figure 11.

In Figure 11, the activation that results from the combined action of prime and target is schematically depicted on the ordinate as a function of perceptual information gathered from the target (on the abscissa). The function is shown for unprimed and primed words (dashed line). The function for primed words was generated by adding a constant to the perceptual information; a larger constant in the left panel (strong prime) than in the right (weak prime). The letter $\boldsymbol{A}$ marks a case with a strong prime and a weak target (incomplete perceptual information extracted from the display). Correspondingly, $B$ marks a strong prime and a strong (clearly displayed) target; $C$ marks a weak prime with a weak target; and $D$ marks a weak prime with a strong target.

The force that drives the differential $\mathbf{N} 400$ effect is the difference between the activation levels achieved by primed and unprimed words-these differences have been denoted $\delta_{\mathrm{A}} \ldots \delta_{\mathrm{D}}$. It is immediately apparent that the semantic effect is larger for degraded targets than for undegraded ones $\left(\delta_{\mathrm{A}}>\delta_{\mathrm{B}}\right.$ and $\left.\delta_{\mathrm{C}}>\delta_{\mathrm{D}}\right)$. In other words, priming brings a greater benefit if processing the target is hard (rather than very easy or impossibly difficult). This is a different way of saying that stimulus degradation interacts with priming, which is a common finding in the behavioral priming literature (Becker \& Killion, 1977; Borowsky \& Besner, 1993; Stolz \& Neely, 1995), although the only N400 study to date showed no such interaction (Holcomb, 1993). However, not only the quality of the target matters. A strong prime brings a greater benefit than a weak one, as a comparison of the two panels 

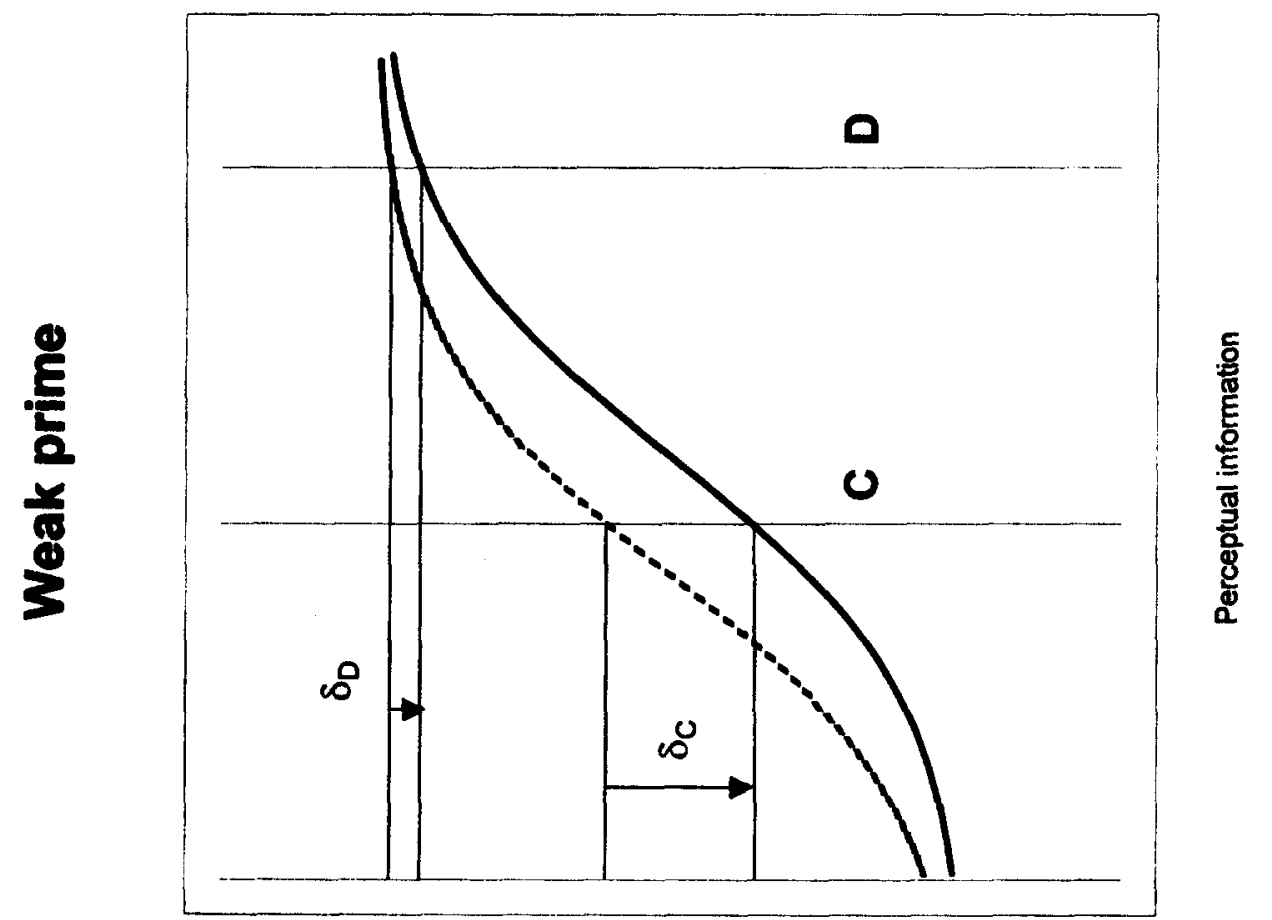

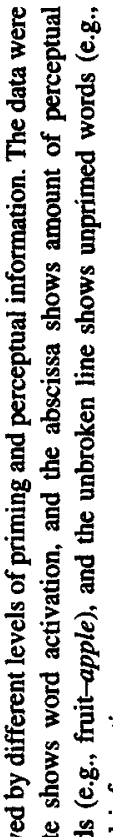

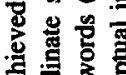

语

홀

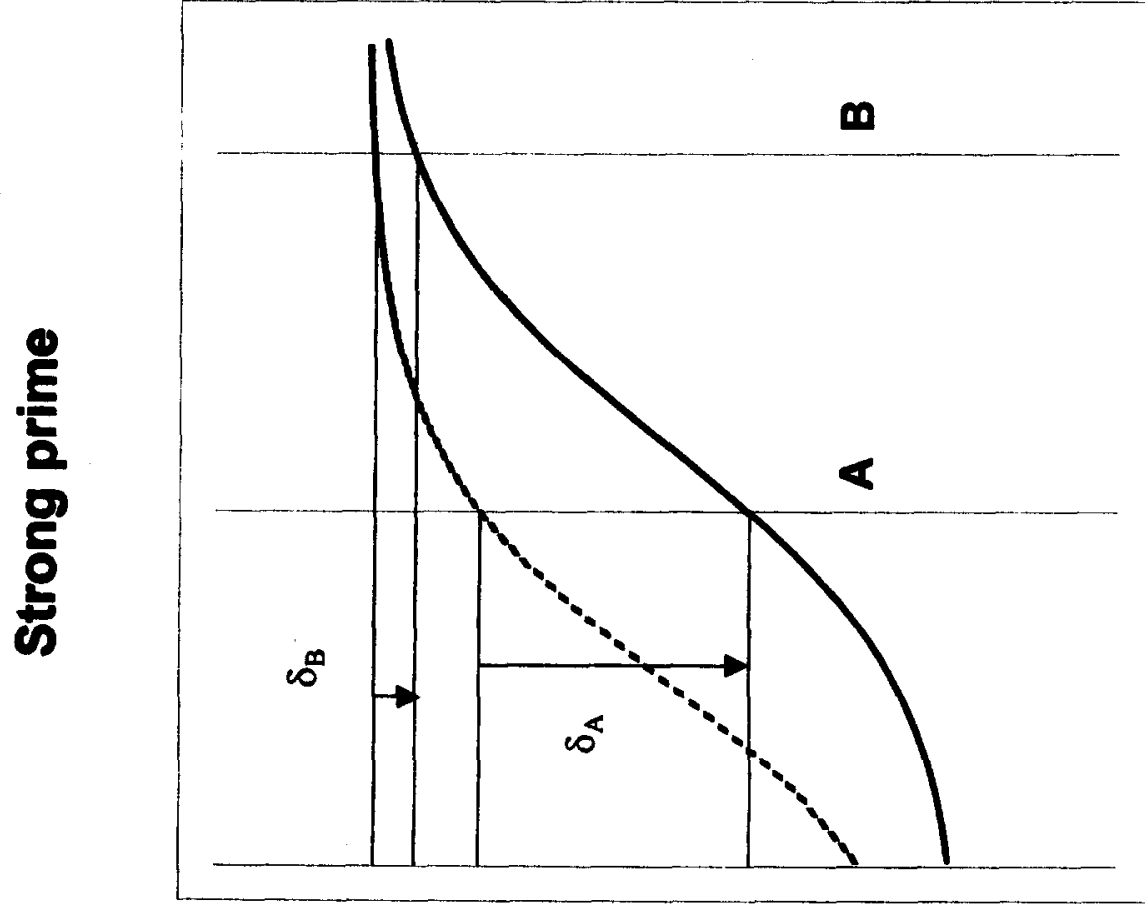

局言合

$\frac{4}{8}$

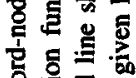

还

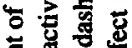

을

을 氕

.

党

응

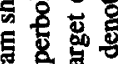

影资

을

要

营紫焉

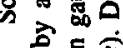

خ 马

in 
confirms. By a "strong" prime, we mean one which is clearly visible (i.e., one whose perceptual information can be fully used); we also mean one that can act through two priming mechanisms rather than just one (i.e., expectancy and spreading activation rather than just the latter).

In sum, the most favorable combination of elements for a semantic effect on word-node activation seems to be a clearly displayed prime, acting over a long SOA on a moderately degraded target. This results in a large semantic differential, $\delta_{A}$, compared with which all other combinations would be less favorable, particularly the combination of weak prime and clear target $\left(\delta_{\mathrm{p}}\right)$. Admittedly, this argument is somewhat schematic, focusing only on word-node activation in lexical access, and leaving out postlexical processes such as integration into context and decision processes. Still, it suffices to show that masking the prime and masking the target may produce different outcomes. Also, it becomes apparent that the present results need not commit us to the view that the processing nature of the $\mathrm{N} 400$ is entirely automatic. On the contrary, controlled processes, such as expectancy (Becker, 1980; Bentin, 1987), in all likelihood play a large part in the build-up before the target display, and only the triggering of this pent-up force is automatic. Indeed, Becker's research has shown that priming between category name and category exemplar allows strong expectancies to develop, a mechanism that accounts for a large part of the RT priming in this paradigm. Using a clearly visible category prime and a long SOA before the masked exemplar word may have worked in favor of allowing both the mechanisms of spreading activation and expectancy to operate.

A consequence of the model presented here is that semantic processing without awareness is more likely to occur for words preceded by semantically related primes than for words without such primes. Although the present data do not speak directly to this issue, the analogous case of primed words being more likely to undergo identification than unprimed ones is demonstrated by identification rates in all three experiments. A similar priming effect on the likelihood of undergoing unconscious processing seems likely, but the issue will have to be addressed directly in experiments before definite conclusions can be drawn.

A puzzling aspect of the results, which is readily apparent in Figure 10 , is the fact that the priming effect size for unidentified words was almost constant across experiments, whereas the effect for identified words differed widely from Experiment 1 (small) to Experiments 2 and 3 (both large). The one change in method likely to account for this difference is in the way the category label was presented: once for each block in Experiment 1 versus once for each trial (and changing between trials) in Experiments 2 and 3. It is not surprising that constantly renewed presentations are more effective as primes than a mere memory trace, maintained by occasional internal repetition. The puzzling aspect is that this made a difference only for words above threshold, not for those below it. Apparently, the two ways of bringing the category to mind induced two different admixtures of controlled and automatic priming processes. The fresh primes may have encouraged generating exemplars, bringing expectancy into play as a priming mechanism (Becker, 1980), whereas blockwise category priming may have relied more on automatic adjustment of activation levels, presumably refreshed periodically by tacit rehearsal of the category cue. However, conjectures about the mechanisms involved are uncertain and await further experimentation.
Some N400 investigations outside the pattern-masking paradigm have indicated semantic access without awareness. Luck et al. (1996) used the attentional blink phenomenon to prevent displayed words from reaching awareness and found the $\mathrm{N400}$ to be reactive to their semantic qualities, despite the fact that they were unreportable only seconds later. Using a different brain potential, Dehaene et al. (1998) have found lateralized readiness potentials to indicate that masked and unidentifiable primes initiate motor preparations for the primed response without awareness.

In a study of repetition priming (Schnyer, Allen, \& Forster, 1997), masked words, which left no episodic memory trace, nevertheless showed repetition effects on ERPs in the N400 time range when repeated after a brief interval. With electrodermal indicators of conditioning, Öhman and Soares (1998) have shown conditioned responses to emotional stimuli to be both learned and subsequently elicited without awareness of the stimulus. Still other physiological measures have recently been applied to the question of unconscious processing. In a PET study of a patient with blindsight, conscious and unconscious perception could be differentiated according to brain activation patterns, with unconscious perception showing more subcortical activity and conscious perception showing more cortical activity (Sahraie et al., 1997). In another PET study (Morris, Ŏhman, \& Dolan, 1998), the amygdala activity evoked by affective faces, to which aversive reactions had been conditioned, showed different lateralization patterns for masked and unmasked displays. Conscious perception evoked activity in the left amygdala and unconscious perception in the right. Thus, in recent years, phenomena of unconscious processing have become approachable through physiological measurements, and important differences between conscious and unconscious processing have been revealed through their patterns of brain activity.

\section{Subjective Identification Criterion}

One feature of the present design that differs from those of many other studies is the use of a concurrent, trial-by-trial measure of conscious perception (cf. Hirshman \& Durante, 1992). The nature of the task allowed a verbal report on the perception, successful or otherwise, of each item, and processing of the dependent variable could be conditionalized on that report. The reason that concurrent verbal reports are often not included is that they are sometimes thought to cause dual-task interference and that they are subject to memory limitations (Fischler \& Goodman, 1978). However, these objections apply primarily to the standard paradigm, where the prime, not the target, is masked. Keeping the prime in working memory can interfere with semantic processing of the target, and the same resource limitations may elbow the prime out of working memory during target processing. In contrast, the present paradigm lets the target be both the subject of semantic processing and the item to be reported.

A concurrent measure of awareness addresses the concerns raised by Holender (1986) and Dark (1988) about the validity of threshold-setting procedures. Dark has objected that retroactive priming may vitiate the standard paradigm by making masked primes more legible when followed by a related word than they are expected to be on the basis of threshold testing with isolated words. 
Although the use of concurrent oral identification avoids the problems associated with a preexperimental threshold determination, it raises an important issue concerning the efficacy of verbal report as a measure of conscious perceptual processing. Framing this question in the terms of Reingold and Merikle (1990), it concerns the way in which the measure satisfies the exclusiveness and exhaustiveness criteria. Although there is no doubt that the exclusiveness criterion is fulfilled (for, almost by definition, a word cannot be reported unless it is consciously experienced), satisfaction of the exhaustiveness criterion must be examined. Potential underreporting by participants who adopt a strict response criterion cannot be a priori excluded, although such a criterion is in conflict with the instruction to guess whenever uncertain. Therefore, additional data were brought to bear on the question.

\section{Qualitative Differences}

With the benefit of physiological data, the issue of qualitative differences between conscious and unconscious processing can be addressed as a question of differences in localization. Recently, explicit and implicit memory have thus been shown to draw on different neural populations, as evidenced by different distributions of ERP activity (Rugg et al., 1998; Schnyer et al., 1997). Because scalp distributions of electrical activity cannot, in the general case, be mapped unequivocally to specific regions in the brain, conclusions about localization drawn from ERP data are limited. However, the cautious inference that different underlying neural populations are active in conditions that give rise to significantly different scalp distributions is adequate for present purposes.

Processing of masked but identified words showed a hemispheric asymmetry, with more positive amplitudes over left parietal areas. In the $\mathrm{N} 400$ time band, this is a frequent finding: "Across several subject samples, we observed a right more negative than left (or left more positive than right) asymmetry in association with words in intermediate sentence positions" (Kutas et al., 1988, p. 228). (The last part of the sentence refers to the fact that typically $\mathrm{N} 400$-provoking, unexpected sentence endings were not necessary to elicit the asymmetry.) Analogously, we saw the left-more-positive-than-right pattern for in-category and out-ofcategory words alike. In contrast to this lateralized pattern, amplitudes for unidentified words were symmetrical. It is tempting to view this in the context of known hemispheric asymmetries in language processing. The specialization for fine-grained language processing in the left hemisphere is abundantly documented, but elementary semantic analysis can take place in the right hemisphere as well (Beeman \& Chiarello, 1998). However, ascribing scalp-recorded potentials to activity in specific brain regions is notoriously difficult. A pattern of larger positive amplitudes on the left side can be ascribed to either a positive component being active on the left side or a counteracting negative component being active on the right side. The cautious inference to be drawn from our data is that identified and unidentified words were processed by partly different neuronal populations. This, in itself, is evidence that different kinds of processing have taken place and argues strongly against the possibility that the processing of unidentified words is just a diluted version of the processing of identified words that is caused by criterion slippage.
The data from Experiment 3 are particularly enlightening, because the pattern of hemispheric symmetry-asymmetry was found in data conditionalized on 6-AFC identification as well. This shows that the symmetric pattern derives not only from the disputed "no man's land" between subjective and objective threshold but also from the deep heartland below objective threshold.

Although raw amplitudes showed this pattern of qualitative differences, the semantic priming effect (i.e., the core N400) showed no significant differences in lateralization pattern. This means that a common core function (e.g., automatic spreading activation) contributes to semantic processing both below and above identification threshold. The question then remains what other processes shape the raw amplitudes for which we have seen qualitative differences. A reasonable guess would be processes in relatively late stages of word identification, such as lexical processing, checking, and contextual integration (assuming that these occur to some extent in parallel with spreading activation). These are controlled processes, for which conscious identification seems to be a prerequisite. They are also clearly candidates for localization into language-specific areas. A potentially illuminating parallel may be the left-parietal recognition effect, found in verbal memory tasks as an enhanced positivity to old, compared with new, words (e.g., Rugg et al., 1998). With roughly the same distribution as our identification effect, the recognition effect may be subserved by some of the same functions.

An entirely different line of reasoning might attempt to explain the identification effects on amplitude asymmetry as a purely perceptual phenomenon. Identified words were, on average, exposed longer than unidentified ones, and perceptual processes were therefore supplied with more and higher quality information. Although this can explain generally larger amplitudes for identified words, some extra assumptions are needed to explain the asymmetry. The left-hemispheric specialization for visual processing of high spatial frequencies could have played a part. Longer exposures could have given more opportunities for the processing of high-frequency visual information (Hellige, 1990; Sergent, 1983), resulting in enhanced left-hemisphere amplitudes. Although this line of events is at least possible, it would imply that the effects would be early and primarily occipital. A test of data from Experiment 2, in which data from left and right occipital as well as left and right parietal electrodes were available, shows that the hemispheric difference was significantly larger over parietal areas than over occipital ones. ${ }^{6}$ The localization of asymmetries over parietal areas indicates a lexical rather than a low-level perceptual origin, as does the late onset time, because the left-over-right asymmetry did not appear before $300 \mathrm{~ms}$ and reached a maximum around $500 \mathrm{~ms}$.

\section{Regression Analysis}

Greenwald et al.'s (1995) regression method is a way of dealing with the difficulty-bordering on impossibility-of demonstrat-

\footnotetext{
${ }^{6}$ Hemispheric differences (P3-P4 and O1-O2) were computed and entered into a two-way ANOVA. The main effect of parietal-occipital position was not reliable, but that of identification was and, critically, the Parietal-Occipital $\times$ Identification interaction, $F(1,29)=19.43$, $M S E=0.90$. For identified words, the parietal difference was 3.39 and the occipital 2.34; for unidentified words, the figures were -0.94 and -0.47 .
} 
ing chance performance on the direct measure for all participants. Taking advantage of the interindividual variability of performance allows the regression analysis to determine, by extrapolation, what performance on the indirect measure would be, had the performance on the direct measure been exactly zero. Provided that the direct measure is otherwise appropriate, this fits the desiderata of the dissociation paradigm: to establish above-zero performance on the indirect measure in the presence of zero performance on the direct measure. By statistically testing the significance of the intercept, it also substitutes the logic of null hypothesis rejection for the much weaker inference of null hypothesis acceptance, associated with earlier demonstrations of zero sensitivity on the direct measure.

In the present study, the role of the direct measure was taken by the binary-choice $d^{\prime}$, whereas the indirect measure was the N400 effect in the semantic task (i.e., the difference between brain responses to in-category and out-of-category words). The intercept proved to be above zero, showing by extension that there would be an $\mathrm{N} 400$ effect, even if performance on the semantic binary-choice were at zero. The result bolsters the case already supported by qualitative differences, viz. that the semantic $\mathrm{N} 400$ effect for unidentified words reflects unconscious processing.

The domain of applicability for the regression method has recently been discussed in comments to the Draine and Greenwald (1998) article by Dosher (1998) and Merikle and Reingold (1998). Merikle and Reingold proposed three conditions that should be met before the conclusion of unconscious perception is justified. Direct and indirect measures should (a) be based on the same metric, (b) index the same perceptual discrimination, and (c) be administered under the same conditions.

As applied to our experiments, we submit that the last two conditions were fulfilled, possibly to a greater extent than in the original Greenwald et al. $(1995,1996)$ studies. The direct measure (binary categorization) and the indirect measure (the N400) are both registered at the same time and in the same trials. The instructions are the same, and the processing giving rise to the behavioral and the ERP responses are indistinguishable for the participants themselves. We see no way that the direct and the indirect measures can be thought of as arising from discriminations differing in difficulty, or resulting from different strategies on the part of the participants.

The requirement that the same metric be used for both measures is, however, not met in our study. Whereas Greenwald and colleagues used $d^{\prime}$ as the metric for both direct and indirect measure, our direct measure was based on $d^{\prime}$, but our indirect measure was a difference (in microvolts) between in-category and out-ofcategory words. We reasoned, however, that the same metric was not necessary, provided that both scales have rational zero points with the same interpretation (i.e., null sensitivity). For both the direct and the indirect measure, a value of zero indicates that no discrimination is being made between words belonging to, and words deviating from, the category. Conversely, points above zero indicate some degree of successful discrimination. For present purposes, intercepts reliably above zero are evidence that ERPs discriminated on the basis of category, when the behavioral response did not.

Dosher (1998) and Klauer, Greenwald, and Draine (1998) have raised a different point concerning the measurements. A theoretical assumption underlying regression calculations is that the predictor is without measurement error. In most practical applications, that assumption is routinely violated with no great consequence. The result is a flattening of the regression line, which, for a positive slope and predictor values above zero, means that the intercept will be overestimated. Because the intercept is normally not of interest, the issue does not become a problem. However, if the intercept is the focus of interest, the issue deserves attention. Overestimation of the intercept occurs to the same extent that the slope is large and positive. In the present data sets, slopes were positive but in no case significantly different from zero. In the four data sets (one each in the first 2 experiments and two in the last), slopes ranged from 0.5 to 1.8 (microvolts per $d^{\prime}$ unit). Correlations were .12, .06 , .24 , and .18 and in no case significant. The findings of null correlations must, however, be tempered by the realization that the power to reject null hypotheses was low.

The absence of a strong relation between direct and indirect measure is both a boon and a curse. A flat slope (normally associated with a weak correlation) safeguards against overestimation of the intercept. On the other hand, a strong correlation between direct and indirect measures could be very persuasive in establishing that they both index the same perceptual process. For our data, as well as for those of Draine and Greenwald (1998), correlations were low, and the comparability of direct and indirect measures therefore needs to be supported on a different basis. The misgivings of Dosher (1998) and Merikle and Reingold (1998) about the regression method are to a large extent concerned with the absence of a (statistical) relation between the two measures. The problem has two aspects. One concerns the intercept itself. If the correlation between the two measures is zero, the intercept simply estimates the mean of the indirect measure. Then, if the group of participants comprises some individuals with high scores on the direct measure, the mean will be unduly affected by these participants, who have shown a high degree of stimulus awareness.

In our whole set of data, the most extreme value on the direct measure was 1.1 . Averages of the $d^{\prime}$ variable were $0.07,0.17,0.23$, and 0.03 , and the 75th percentiles in the four sets were situated at $0.34,0.31,0.40$, and 0.24 . Thus, very few data points, if any, could be said to belong to participants performing substantial conscious discrimination,

The other aspect of the problem is more conceptual than statistical. If there is no correlation between measures, the question can be raised as to whether they refer to the same perceptual process. We agree with the point made by Greenwald and Draine (1998) that independence of the processes underlying conscious and unconscious perception, even faced with the same task, is both possible and probable. Greenwald and Draine gave the example of blindsight, where separate anatomical pathways form the likely basis of independent processes. In our data, some degree of independence that was due to method variance was to be expected between data as different as electrophysiological activation and behavioral responses. This partial independence need not detract from the fact that both measures reflect the same semantic discrimination, made at the same time and under the same circumstances.

The final and crucial stumbling block has been the exhaustiveness criterion, for if the direct measure does not embrace the full range of relevant conscious processing, a significant intercept in the regression equation will not convincingly demonstrate that unconscious processing has taken place. Whereas Merikle and 
Reingold (1998) took a generalized skeptical attitude to the attainment of exhaustiveness, Greenwald and Draine (1998) reserved judgment on this as an empirical issue. We agree that the issue has to be judged on the merits of each experiment per se. In the present case, it is difficult to see how any conscious knowledge, relevant for shaping the semantic response of the brain, could have escaped the direct measure. If a participant sees a word well enough to form a conscious opinion of its semantic category, why-short of deliberate deception-would he or she withhold that knowledge when making a simple yes-no decision about the category?

\section{Objective Threshold}

In the final experiment, identification was made to rely on discriminative performance in a multiple-choice selection (6AFC). When analysis was narrowed down to those trials where an incorrect alternative was chosen, the ERPs retained the capacity to distinguish between words on semantic grounds, with little loss of power. This seems to fulfill the dissociation paradigm's desideratum of an indirect effect without a direct one. The case rests on how well the direct test fulfills the criterion of exhaustiveness. Could the participant form a conscious representation of the displayed word and still fail to pick it out among six alternatives only seconds later? We find it very unlikely, apart from trivial errors (such as slipping with the mouse, or misreading the decimated, every-second-letter alternatives). Such errors could just as easily arise with clearly identifiable words, and the data on verbally identified words showed that they occur with a frequency of $0.5 \%$. Other than that, performance on the 6-AFC test should cover the whole field of conscious, task-relevant knowledge (and a certain amount of task-irrelevant knowledge as well, such as knowing that one letter of the word was an $r$, when the task was to decide whether it refers to a mammal).

In summary, we used three types of measures, with each being sensitive to semantic processing in a different way. Verbal report was the least sensitive, covering only conscious processing, and possibly not even all of that. Discriminative performance (binary choice or $6-A F C$ ) proved to be a more sensitive measure than verbal report here, as in many earlier studies. It encompasses all of conscious processing, and something else in addition. Unconscious semantic processing may make its end result available to awareness, where it can influence choice among given alternatives. Because the experience of processing is lacking, the choice response is usually given with low confidence. Our third type of measure was the psychophysiological response, which showed a particular kind of sensitivity. When the other two types were reduced to null accuracy, ERPs could still discriminate between words on the basis of their category membership. However, with other sets of measures, behavioral variables can sometimes show greater sensitivity than ERPs. Thus, with masked primes and unmasked targets, rather than the reverse, Brown and Hagoort (1993) found effects of priming on reaction time but not on the N400.

In our experiments, both semantic discriminative responding (the yes-no category choice) and ERPs could be said to show signs of unconscious processing, but they appeared to be relatively independent of each other. The semantic binary choice and the N400 were not significantly correlated across participants in any of our three experiments. However, conclusions about independence must be restrained by considerations of statistical power. Accepting a conclusion of independent processes would require a greater number of participants than we had.

When considering processing below objective threshold (i.e., trials with erroneous responses in the 6-AFC of Experiment 3), we found that semantic binary choice showed only chance-level discrimination, whereas ERPs showed significant discriminatory power. Thus, with our set of measures, the psychophysiological response proved to be the most sensitive indicator of unconscious processing.

In conclusion, we have found evidence of semantic processing of words that escaped conscious identification. By any of several definitions of awareness, words were processed semantically without awareness. Moreover, there was evidence that conscious and unconscious word-activation processes are qualitatively different, in that they recruit neural populations with partly different spatial distributions in the brain.

\section{References}

Adams, J. K. (1957). Laboratory studies of behaviour without awareness. Psychological Bulletin, 54, 383-405.

Allport, A. (1977). On knowing the meaning of words we are unable to report. In S. Dornic (Ed.), Attention and performance (Vol. VI). Hillsdale, NJ: Erlbaum.

Anderson, J. E., \& Holcomb, P. J. (1995). Auditory and visual semantic priming using different stimulus onset asynchronies: An event-related brain potential study. Psychophysiology, 32, 177-190.

Balota, D. A. (1983). Automatic semantic activation and episodic memory encoding. Journal of Verbal Learning and Verbal Behavior, 22, 88-104.

Barrett, S. E., \& Rugg, M. D. (1990). Event-related potentials and the semantic matching of pictures. Brain \& Cognition, 14, 201-212.

Becker, C. A. (1980). Semantic context effects in visual word recognition: An analysis of semantic strategies. Memory and Cognition, 8, 493-512.

Becker, C. A., \& Killion, T. H. (1977). Interaction of visual and cognitive effects in word recognition. Joumal of Experimental Psychology: Human Perception and Performance, 3, 389-401.

Beeman, M., \& Chiarello, C. (1998). Complementary right- and lefthemisphere language comprehension. Current Directions in Psychological Science, 7.

Bentin, S. (1987). Event-related potentials, semantic processes, and expectancy factors in word recognition. Brain \& Language, 31, 308-327.

Bentin, S., McCarthy, G., \& Wood, C. C. (1985). Event-related potentials, lexical decision and semantic priming. Electroencephalography and Clinical Neurophysiology, 60, 343-355.

Besson, M., Fischler, I., Boaz, T., \& Raney, G. (1992). Effects of automatic associative activation on explicit and implicit memory tests. Journal of Experimental Psychology: Leaming, Memory, \& Cognition, 18, 89105.

Boddy, J. (1986). Event-related potentials in chronometric analysis of primed word recognition with different stimulus onset asynchronies. Psychophysiology, 23, 232-245.

Borowsky, R., \& Besner, D. (1993). Visual word recognition: A multistage activation model. Journal of Experimental Psychology: Learning, Memory and Cognition, 19, 813-840.

Brown, C., \& Hagoort, P. (1993). The processing nature of the N400: Evidence from masked priming. Joumal of Cognitive Neuroscience, 5 , 34-44.

Brualla, J., Romero, M. F., Serrano, M., \& Valdizan, J. R. (1998). Auditory event-related potentials to semantic priming during sleep. Electroencephalography and Clinical Neurophysiology, 108, 283-290.

Cheesman, J., \& Merikle, P. M. (1985). Word recognition and consciousness. In D. Besner, T. G. Waller, \& G. E. MacKinnon (Eds.), Reading 
research: Advances in theory and practice (Vol. 5, pp. 311-352). New York: Academic Press.

Cheesman, J., \& Merikle, P. M. (1986). Distinguishing conscious from unconscious perceptual processes. Canadian Journal of Psychology, 40, 343-367.

Chwilla, D. J., Brown, C. M., \& Hagoort, P. (1995). The N400 as a function of the level of processing. Psychophysiology, 32, 274-285.

Collins, A. M., \& Loftus, E. F. (1975). A spreading-activation theory of semantic processing. Psychological Review, 82, 407-428.

Dark, V. J. (1988). Semantic priming, prime reportability, and retroactive priming are interdependent. Memory \& Cognition, 16, 299-308.

Dehaene, S., Naccache, L., Le Clec, H. G., Koechlin, E., Mueller, M., Dehaene Lambertz, G., van de Moortele, P. F., \& Le Bihan, D. (1998). Imaging unconscious semantic priming. Nature, 395, 597-600.

Dixon, N. F. (1971). Subliminal perception. London: McGraw-Hill.

Dosher, B. A. (1998). The response-window regression method-Some problematic assumptions: Comment on Draine and Greenwald (1998). Journal of Experimental Psychology: General, 127, 311-317.

Draine, S., \& Greenwald, A. G. (1998). Replicable unconscious semantic priming. Journal of Experimental Psychology: General, 127, 286-303.

Eriksen, C. W. (1960). Discrimination and learning without awareness: A methodological survey and evaluation. Psychological Review, 67, 279300.

Fischler, I., \& Goodman, G. O. (1978). Latency of associative activation in memory. Journal of Experimental Psychology: Human Perception and Performance, 4, 455-470.

Fischler, I., \& Raney, G. E. (1991). Language by eye: Behavioral and psychophysiological approaches to reading. In R. J. Jennings \& M. G. H. Coles (Eds.), Handbook of cognitive psychophysiology: Central and autonomic nervous system approaches (pp. 511-574). New York: Wiley.

Fowler, C. A., Wolford, G., Slade, R., \& Tassinary, L. (1981). Lexical access with and without awareness. Journal of Experimental Psychology: General, 110, 341-362.

Green, D. M., \& Swets, J. A. (1974). Signal detection theory and psychophysics. Huntington, NY: Krieger.

Greenwald, A. G., \& Draine, S. C. (1998). Distinguishing unconscious from conscious cognition-Reasonable assumptions and replicable findings: Reply to Merikle and Reingold (1998) and Dosher (1998). Journal of Experimental Psychology: General, 127, 320-324.

Greenwald, A. G., Draine, S. C., \& Abrams, R. L. (1996). Three cognitive markers of unconscious semantic activation. Science, 273, 1699-1702.

Greenwald, A. G., Klinger, M. R., \& Schuh, E. S. (1995). Activation by marginally perceptible ("subliminal") stimuli: Dissociation of unconscious from conscious cognition. Journal of Experimental Psychology: General, 124, 22-42.

Hellige, J. (1990). Hemispheric asymmetry. Annual Review of Psychology, 41, 55-80.

Hirshman, E., \& Durante, R. (1992). Prime identification and semantic priming. Journal of Experimental Psychology: Learning, Memory, and Cognition, 18, 255-265.

Holcomb, P. J. (1988). Automatic and attentional processing: An eventrelated brain potential analysis of semantic priming. Brain \& Language, 35, 66-85.

Holcomb, P. J. (1993). Semantic priming and stimulus degradation: Implications for the role of the $\mathrm{N} 400$ in language processing. Psychophysiology, 30, 47-61.

Holcomb, P. J., \& McPherson, W. B. (1994). Event-related brain potentials reflect semantic priming in an object decision task. Brain \& Cognition, 24, 259-276.

Holender, D. (1986). Semantic activation without conscious identification in dichotic listening, parafoveal vision, and visual masking: A survey and appraisal. Behavioral and Brain Sciences, 9, 1-66.

Klauer, K. C., Greenwald, A. G., \& Draine, S. C. (1998). Correcting for measurement error in detecting unconscious cognition: Comment on Draine and Greenwald (1998). Journal of Experimental Psychology: General, 127, 318-319.

Kounios, J., \& Holcomb, P. J. (1994). Concreteness effects in semantic processing: ERP evidence supporting dual-coding theory. Journal of Experimental Psychology: Learning, Memory, \& Cognition, 20, 804823.

Kutas, M., \& Hillyard, S. A. (1980). Reading senseless sentences: Brain potentials reflect semantic incongruity. Science, 207, 203-205.

Kutas, M., \& Hillyard, S. A. (1989). An electrophysiological probe of incidental semantic association. Journal of Cognitive Neuroscience, 1 , $38-49$.

Kutas, M., Van Petten, C., \& Besson, M. (1988). Event-related potential asymmetries during the reading of sentences. Electroencephalography and Clinical Neurophysiology, 69, 218-233.

Luck, S. J., Vogel, E. K., \& Shapiro, K. L. (1996). Word meanings can be accessed but not reported during the attentional blink. Nature, 383 , 616-618.

Marcel, A. (1980). Conscious and preconscious recognition of polysemous words: Locating the selective effects of prior verbal context. In Nickerson (Ed.), Attention and performance (Vol. VIII). Hillsdale, NJ: Erlbaum.

Marcel, A. J. (1983a). Conscious and unconscious perception: An approach to the relations between phenomenal experience and perceptual processes. Cognitive Psychology, 15, 238-300.

Marcel, A. J. (1983b). Conscious and unconscious perception: Experiments on visual masking and word recognition. Cognitive Psychology, 15, 197-237.

Marcel, A., \& Patterson, K. (1978). Word recognition and production: Reciprocity in clinical and normal studies. In J. Requin (Ed.), Attention and performance (Vol. VII). Hillsdale, NJ: Erlbaum.

McCarthy, G., \& Nobre, A. C. (1993). Modulation of semantic processing by spatial selective attention. Electroencephalography and Clinical Neurophysiology, 88, 210-219.

McCarthy, G., \& Wood, C. C. (1985). Scalp distributions of event-related potentials: An ambiguity associated with analysis of variance models. Electroencephalography and Clinical Neurophysiology, 62, 203-208.

Merikle, P. M., \& Reingold, E. M. (1990). Recognition and lexical decision without detection-Unconscious perception. Journal of Experimental Psychology: Human Perception and Performance, 16, 574-583.

Merikle, P. M., \& Reingold, E. M. (1992). Measuring unconscious perceptual processes. In R. F. Bornstein \& T. S. Pittman (Eds.), Perception without awareness: Cognitive, clinical and social perspectives (pp. 55-80). New York: Guilford.

Merikle, P. M., \& Reingold, E. M. (1998). On demonstrating unconscious perception: Comment on Draine and Greenwald (1998). Journal of Experimental Psychology: General, 127, 304-310.

Morris, J. S., Öhman, A., \& Dolan, R. J. (1998). Conscious and unconscious emotional learning in the human amygdala. Nature, 393, 467470.

Morton, J. (1969). Interaction of information in word recognition. Psychological Review, 76, 165-178.

Münte, T. F., \& Heinze, H. J. (1991). Aktivierung des semantischen Speichers durch unterschwellige Wortstimuli? Eine Analyse mit erregniskorrelierten Hirnpotentialen [Activation of the semantic memory by subliminal word stimuli? An analysis using event-related potentials]. Zeitschrift für EEG und EMG, 22, 3-9.

Neely, J. H. (1991). Semantic priming effects in visual word recognition: A selective review of current findings and theories. In D. Besner \& G, Humphreys (Eds.), Basic processes in reading. Hillsdale, NJ: Erlbaum.

Neely, J. H., \& Keefe, D. E. (1989). Semantic context effects on visual word-processing-A hybrid prospective retrospective processing theory. Psychology of Learning and Motivation. Advances in Research and Theory, 24, 207-248. 
Neville, H. J., Coffey, S. A., Lawson, D. S., Fischer, A., Emmorey, K., \& Bellugi, U. (1997). Neural systerns mediating American sign language: Effects of sensory experience and age of acquisition. Brain Language, 57, 285-308.

Nigam, A., Hoffman, J. E., \& Simons, R. F. (1992). N400 to semantically anomalous pictures and words. Journal of Cognitive Neuroscience, 4, 15-22.

Ohman, A., \& Soares, J. (1998). Emotional conditioning to masked stimuli: Expectancies for aversive outcomes following nonrecognized fearrelevant stimuli. Journal of Experimental Psychology: General, 127, 69-82.

Osterhout, L., \& Holcomb, P. J. (1995). Event-related potentials and language comprehension. In M. D. Rugg \& M. G. H. Coles (Eds.), Electrophysiology of mind: Event-related brain potentials and cognition. Oxford, England: Oxford University Press.

Polich, J. (1985). Semantic categorization and event-related potentials. Brain and Language, 26, 304-321.

Reingold, E. M., \& Merikle, P. M. (1990). On the inter-relatedness of theory and measurement in the study of unconscious processes. Mind and Language, 5, 9-28.

Rugg, M. D., Mark, R. E., Walla, P., Schloerscheidt, A. M., Birch, C. S., \& Allan, K. (1998). Dissociation of the neural correlates of implicit and explicit memory. Nature, 392, 595-598.

Sahraie, A., Weiskrantz, L., Barbur, J. L., Simmons, A., Williams, S. C. R., \& Brammer, M. J. (1997, August). Pattern of neuronal activity associated with conscious and unconscious processing of visual signals. Proceedings of the National Academy of Sciences, USA, 94, 9406-9411.
Schnyer, D. M., Allen, J. J. B., \& Forster, K. I. (1997). Event-related brain potential examination of implicit memory processes: Masked and unmasked repetition priming. Neuropsychology, 11, 243-260.

Sergent, J. (1983). Role of the input in visual hemispheric asymmetries. Psychological Bulletin, 93, 481-512.

Smith, M. C., Besner, D., \& Miyoshi, H. (1994). New limits to automaticity: Context modulates semantic priming. Journal of Experimental Psychology: Learning, Memory and Cognition, 20, 104-115.

Stolz, J., \& Besner, D. (1997). Visual word recognition: Effort after meaning but not (necessarily) meaning after effort. Journal of Experimental Psychology: Human Perception and Performance, 23, 13141322.

Stolz, J. A., \& Neely, J. H. (1995). When target degradation does and does not enhance semantic context effects in word recognition. Journal of Experimental Psychology: Learning, Memory, and Cognition, 21, 596611.

Van Petten, C., \& Kutas, M. (1990). Interactions between sentence context and word frequency in event-related brain potentials. Memory \& Cognition, 18, 380-393.

Young, M. P., \& Rugg, M. D. (1992). Word frequency and multiple repetition as determinants of the modulation of event-related potentials in a semantic classification task. Psychophysiology, 29, 664676.
Received April 23, 1999

Revision received January 31, 2000

Accepted February 1, 2000 